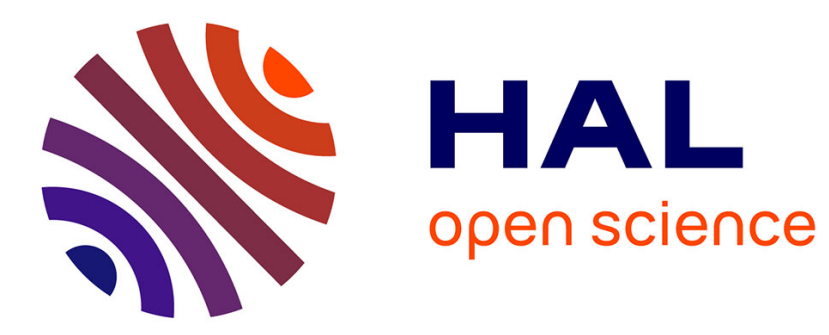

\title{
Dynamics of pulsatile flow through model abdominal aortic aneurysms
}

\author{
Shyam Sunder Gopalakrishnan, Benoît Pier, Arie Biesheuvel
}

\section{To cite this version:}

Shyam Sunder Gopalakrishnan, Benoît Pier, Arie Biesheuvel. Dynamics of pulsatile flow through model abdominal aortic aneurysms. Journal of Fluid Mechanics, 2014, 758, pp.150 - 179. 10.1017/jfm.2014.535 . hal-01084537

\section{HAL Id: hal-01084537 https://hal.science/hal-01084537}

Submitted on 19 Nov 2014

HAL is a multi-disciplinary open access archive for the deposit and dissemination of scientific research documents, whether they are published or not. The documents may come from teaching and research institutions in France or abroad, or from public or private research centers.
L'archive ouverte pluridisciplinaire HAL, est destinée au dépôt et à la diffusion de documents scientifiques de niveau recherche, publiés ou non, émanant des établissements d'enseignement et de recherche français ou étrangers, des laboratoires publics ou privés. 


\title{
Dynamics of pulsatile flow through model abdominal aortic aneurysms
}

\author{
Shyam Sunder Gopalakrishnan ${ }^{1} \uparrow,{\text { Benoît } \text { Pier }^{1} \text { and Arie Biesheuvel }}^{1}$ \\ ${ }^{1}$ Laboratoire de mécanique des fluides et d'acoustique, CNRS - École centrale de Lyon - Université \\ Claude-Bernard Lyon 1 - INSA Lyon, 36 avenue Guy-de-Collongue, F-69134 Écully, France
}

(Received 9 April 2014; revised 8 July 2014; accepted 9 September 2014; first published online 7 October 2014)

To contribute to the understanding of flow phenomena in abdominal aortic aneurysms, numerical computations of pulsatile flows through aneurysm models and a stability analysis of these flows were carried out. The volume flow rate waveforms into the aneurysms were based on measurements of these waveforms, under rest and exercise conditions, of patients suffering abdominal aortic aneurysms. The Reynolds number and Womersley number, the dimensionless quantities that characterize the flow, were varied within the physiologically relevant range, and the two geometric quantities that characterize the model aneurysm were varied to assess the influence of the length and maximal diameter of an aneurysm on the details of the flow. The computed flow phenomena and the induced wall shear stress distributions agree well with what was found in PIV measurements by Salsac et al. (J. Fluid Mech., vol. 560, 2006, pp. 19-51). The results suggest that long aneurysms are less pathological than short ones, and that patients with an abdominal aortic aneurysm are better to avoid physical exercise. The pulsatile flows were found to be unstable to three-dimensional disturbances if the aneurysm was sufficiently localized or had a sufficiently large maximal diameter, even for flow conditions during rest. The abdominal aortic aneurysm can be viewed as acting like a 'wavemaker' that induces disturbed flow conditions in healthy segments of the arterial system far downstream of the aneurysm; this may be related to the fact that one-fifth of the larger abdominal aortic aneurysms are found to extend into the common iliac arteries. Finally, we report a remarkable sensitivity of the wall shear stress distribution and the growth rate of three-dimensional disturbances to small details of the aneurysm geometry near the proximal end. These findings suggest that a sensitivity analysis is appropriate when a patient-specific computational study is carried out to obtain a quantitative description of the wall shear stress distribution.

Key words: biological fluid dynamics, instability

\section{Introduction}

An abdominal aortic aneurysm (figure 1) is a localized dilatation of the infrarenal aortic wall, between the renal arteries and the iliac bifurcation. A dilatation of the aorta is considered to be an aneurysm when its maximal diameter is greater

$\dagger$ Email address for correspondence: shyam-sunder.gopalakrishnan@univ-lyon1.fr 
(a)

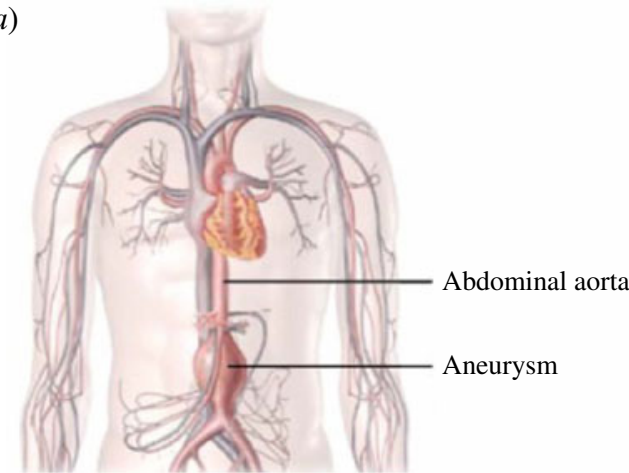

(b)

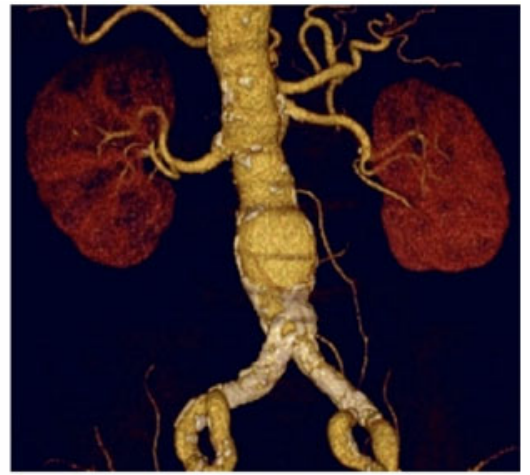

FIGURE 1. (Colour online) (a) Sketch of an abdominal aortic aneurysm that is confined to the infrarenal aorta (Lasheras 2007). (b) Image of an abdominal aortic aneurysm observed in vivo (courtesy: P. Feugier, Hôpital Édouard-Herriot).

than 1.5 times the local diameter of the healthy aorta (Johnston et al. 1991), which is approximately $2 \mathrm{~cm}$ in the abdominal parts of the aorta. One-fifth of large abdominal aneurysms are not limited to the infrarenal aorta, but also extend into one or both of the common iliac arteries (Armon et al. 1998).

When the mechanical stress in the vessel wall exceeds a critical value the dilated vessel ruptures, which leads to a bleeding that is often lethal. Presently, clinical intervention is recommended if the maximum diameter reaches $5.0 \mathrm{~cm}$ in women and $5.5 \mathrm{~cm}$ in men or if the maximal diameter increases by more than $0.5-1 \mathrm{~cm}$ in one year (Grootenboer et al. 2009). Yet, as observed by Vorp (2007), many smaller lesions rupture $(13 \%$ of those of less than $5 \mathrm{~cm}$ ), while larger lesions may not rupture over long periods ( $54 \%$ of those of over $7 \mathrm{~cm}$ ). This leaves physicians to face the dilemma of either subjecting patients to a complex surgery with high morbidity and complications or to an unknown risk of rupture, to paraphrase Lasheras (2007). As pointed out by Humphrey \& Taylor (2008) and Humphrey \& Holzapfel (2012), there is a pressing need to better understand the mechanobiology, pathophysiology and treatment of abdominal aortic aneurysm; an understanding that should result from combining advances in vascular biology, medical imaging, biofluid mechanics and biosolid mechanics.

To provide a background and motivation for the present study, we briefly mention some of the recent work on the haemodynamics of abdominal aortic aneurysms; for a more elaborate discussion the reader is referred to the reviews by Lasheras (2007) and Humphrey \& Taylor (2008). A highly advanced approach, which has increasingly become the standard, is the experimental and computational study of blood flow in models of the cardiovascular system obtained from patient-specific anatomical data acquired by medical imaging. An introduction to this approach can be found in Taylor \& Draney (2004) and Taylor \& Figueroa (2009). Recent studies of the haemodynamics of abdominal aortic aneurysms using this approach include Les et al. (2010), Sheidaei et al. (2011), Stamatopoulos et al. (2011) and Suh et al. (2011). Such studies can provide a quantitative description of the flow in an aneurysm and the distribution of shear stress along the vessel wall, and may even incorporate the presence of atherosclerotic plaque. There is no question that in the near future these computational tools will be sufficiently developed that they will be important for decisions on medical intervention. Yet, given the complexity of the geometries used 
in these studies, it will prove very difficult to develop an understanding of the fluid mechanical phenomena that occur and of the relation between these phenomena and the geometrical details. It seems that there will remain a need for detailed studies of the flow in simplified 'unrealistic' models of aneurysms. This paper presents a study of pulsatile (physiological) flow through an axisymmetric model aneurysm with a wall that is described by a Gaussian function. In doing so, we follow, to a certain extent, the 'simplified approach' of the work by Taylor \& Yamaguchi (1994), Finol, Keyhani \& Amon (2002), Yip \& Yu (2002), Salsac, Sparks \& Lasheras (2004), Deplano et al. (2007) and Sheard (2009). Regarding the assumption of an axisymmetric geometry, it may be added that it has been observed that aneurysms tend to be symmetric during the early stages of the disease, only becoming non-axisymmetric during the later stages, as reported in Salsac (2005). This means that our results may have a direct bearing on what can be observed during the early stages. To this we should add that in our work we focus on the fluid flow dynamics and assume rigid boundaries, ignoring any compliance of the arterial wall or of an endoluminal thrombus. The idealized geometry used in this study should be understood as the boundaries of the fluid domain (where the blood is in contact either with the arterial wall or with a thrombus).

A question that has received some attention in recent years is the connection between cardiovascular flow and conditions of physical activity, in particular the differences between the conditions of rest and exercise. Here, the articles by Egelhoff et al. (1999), Taylor, Hughes \& Zarins (1999), Deplano et al. (2007), Les et al. (2010) and Suh et al. (2011) should be mentioned. It has been hypothesized that prolonged physical exercise may eventually slow the growth of aneurysms, but supporting arguments are indecisive. What has been established is that during part of the cardiac cycle the blood flow in the abdominal aorta becomes weakly turbulent during exercise, but it remains laminar during rest conditions (Les et al. 2010). A local dilatation of the abdominal aorta may be expected to promote instability of the blood flow, and it is not unlikely that flashes of turbulence may occur during part of the cardiac cycle even during rest conditions (Yip \& Yu 2001). This is believed to be beneficial, on the grounds that the presence of turbulence reduces the size of regions of flow stasis and the existence of a correlation between the presence of such regions and thrombus formation (Reininger et al. 1994; Vorp et al. 2001; Salsac et al. 2004). Although we have no support for this, we believe that the repeated occurrence of a vessel wall loading with random small-scale fluctuations in time and space, due to the fact that during part of the cardiac cycle the flow becomes turbulent, may have a detrimental effect on the structure of the vessel wall, and thereby enhance the growth and rupture of aneurysms. To the best of our knowledge, a study of the stability of flow through abdominal aneurysms, even using a simple model configuration, has not yet been published. The present paper provides a first look into this complex problem.

The paper is organized as follows. The geometry of the model aneurysm, the parameters and structure of the pulsatile flow at the inlet of the aneurysm, as well as the numerical methods used to analyze the flow in the aneurysm are described in $\S 2$. It will appear that two geometrical dimensionless quantities, representative of the length and the maximum diameter of the dilatation, and two dimensionless physical flow quantities, the Reynolds number and the Womersley number, characterize the problem. The results of numerical computations of the flow through the model aneurysm are presented in $\S 3$, where we also discuss how the characteristics of this flow change as the geometrical and physical parameters vary in a physiologically 


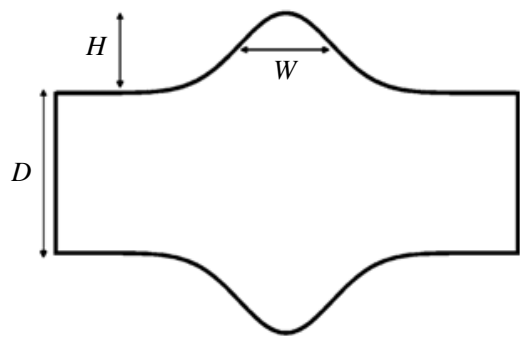

FIGURE 2. The Gaussian vessel wall configuration used in the present study. In this figure $W / D=0.5, H / D=0.5$.

realistic range. Most of the discussion is centred on a blood flow rate waveform that is observed during rest, with a brief discussion of what has been found for a flow rate waveform that is typical for an exercise condition. Section 4 describes the first results of a global stability analysis of pulsatile flow through a model aneurysm, and how these results change with the values of the geometrical and dynamical flow parameters. In $\S 5$ we report a remarkable sensitivity of the flow to details of the aneurysm geometry. Finally, §6 summarizes the main conclusions. Some physiological implications are mentioned, even though these are highly speculative.

\section{Methodology}

\subsection{Geometry}

The axisymmetric model dilatation that is considered in the present study is shown in figure 2. The vessel wall is described by a Gaussian function

$$
r(z)=\left[\frac{D}{2}+H \exp \left(-\frac{z^{2}}{2 W^{2}}\right)\right],
$$

where $z$ and $r$ denote the axial and radial coordinates with the origin taken at the centre of the dilatation. Thus, the geometrical quantities that characterize the model aneurysm are the inlet diameter $D$, the height $H$ and the width $W$. The length of an aneurysm depends on how it is defined; in most definitions the length will involve both parameters $H$ and $W$. The so-called dilatation ratio $D_{R}$, the ratio of the maximum diameter to the inlet diameter, is here given by $D_{R}=1+2 H / D$. In what follows, lengths are non-dimensionalized by the inlet diameter $D$, so that the model aneurysm is characterized by two geometrical dimensionless quantities $H$ and $W$. To study the effects of variations in the size of aneurysms or, to put it differently, the change in the hydrodynamic loading of the vessel wall at different stages of the development of an aneurysm, two values of $W$ are chosen, 0.5 and 1 , and $H$ is varied in the range $0.3 \leqslant H / D \leqslant 1$ (this corresponds to $1.6 \leqslant D_{R} \leqslant 3$ ). Most of the geometrical parameters considered in the present study correspond to limited size aneurysms (except for the case where $H=1$ ) which are clinically shown to be devoid of an endoluminal thrombus (Harter et al. 1982).

\subsection{Fluid flow}

The velocity distribution of the unperturbed blood flow, taken to be a Newtonian fluid, is considered as solenoidal and axisymmetric, with zero velocity in the azimuthal 
direction. The governing equations in cylindrical coordinates are then

$$
\begin{gathered}
\frac{\partial U_{r}}{\partial t}+\left(U_{r} \frac{\partial U_{r}}{\partial r}+U_{z} \frac{\partial U_{r}}{\partial z}\right)=-\frac{\partial P}{\partial r}+\frac{1}{R e}\left[\frac{1}{r} \frac{\partial}{\partial r}\left(r \frac{\partial U_{r}}{\partial r}\right)+\frac{\partial^{2} U_{r}}{\partial z^{2}}-\frac{U_{r}}{r^{2}}\right], \\
\frac{\partial U_{z}}{\partial t}+\left(U_{r} \frac{\partial U_{z}}{\partial r}+U_{z} \frac{\partial U_{z}}{\partial z}\right)=-\frac{\partial P}{\partial z}+\frac{1}{R e}\left[\frac{1}{r} \frac{\partial}{\partial r}\left(r \frac{\partial U_{z}}{\partial r}\right)+\frac{\partial^{2} U_{z}}{\partial z^{2}}\right], \\
\frac{\partial U_{r}}{\partial r}+\frac{U_{r}}{r}+\frac{\partial U_{z}}{\partial z}=0,
\end{gathered}
$$

where $U_{r}(r, z)$ and $U_{z}(r, z)$ are the radial and axial velocity components, respectively, and $P(r, z)$ is the pressure distribution. The Reynolds number $R e$ is based on the vessel diameter and the mean flow velocity, $R e=4 \bar{Q} / \pi D v$, with $\bar{Q}$ the volume flow rate averaged over a cardiac pulse cycle and $v$ the kinematic viscosity of the fluid. According to $\mathrm{Ku}$ (1997), the peak Reynolds number, based on the maximum volume flow rate during a cycle, can vary from 600 at rest up to 6000 under exercise conditions in the abdominal aorta.

The governing equations have been solved imposing the no-slip condition at the vessel wall, assumed to be rigid, a standard no-stress condition at the outlet of the vessel, and the usual symmetry conditions at the axis. The inlet velocity distribution is the Womersley solution for the time-dependent flow in a cylindrical vessel of constant circular cross-section (Pedley 1979). This velocity distribution is determined completely by specifying the radian frequency $\omega$ and the Fourier components $Q_{n}$ of the imposed flow rate waveform,

$$
Q(t)=\sum_{n=-\infty}^{\infty} Q_{n} \mathrm{e}^{\mathrm{i} n \omega t} .
$$

Writing this inlet condition in dimensionless form introduces a second dimensionless physical quantity that characterizes the fluid flow, namely the Womersley number $W o=D(\omega / 4 v)^{1 / 2}$ (Pedley 1979). The two physiological flow rate waveforms that have been used in the present study are discussed in the following section.

\subsection{Physiological flow rate waveforms}

Blood flow rate waveforms differ between rest and exercise conditions. Recently, several studies have been carried out to elucidate how these differences may affect the pathogenesis of abdominal aneurysms. The studies of Salsac et al. (2006) and Sheard (2009) were based on a flow rate waveform obtained from a healthy male subject at rest. The difficulty is that the flow rate waveform varies significantly with the location in the aorta. Moreover, during the progression of the pathology the flow rate waveform may change in response to the alterations of the blood vessel geometry. In our study, we have used data presented in a recent article by Suh et al. (2011), who recorded blood flow rate waveforms of 10 subjects (nine male, one female) suffering from abdominal aortic aneurysms. The recordings were made at an infrarenal location just upstream of the aneurysm, and during both rest and exercise conditions.

The waveforms, the one corresponding to rest conditions denoted $Q 1$ and the one corresponding to exercise conditions denoted $Q 2$, are shown in figure $3(a)$; in figure $3(b)$ the non-dimensionalized value of the mean flow rate $\bar{Q}$ has been given the 

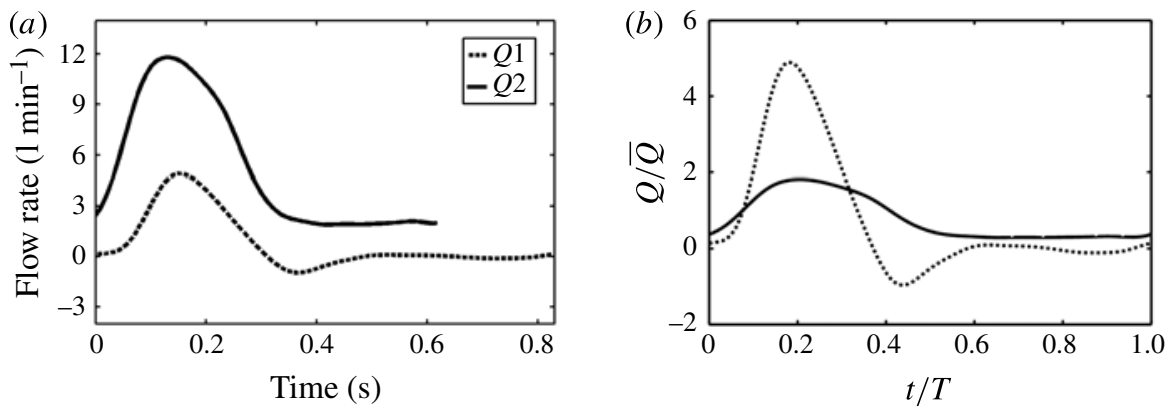

FIGURE 3. (a) Flow rate waveforms corresponding to rest $(Q 1)$ and exercise $(Q 2)$ conditions, as recorded by Suh et al. (2011) at an infrarenal location just above an abdominal aortic aneurysm. (b) The two flow rate waveforms when non-dimensionalized by the cycle-averaged volume flow rate.

$\begin{array}{lcccccc}\text { Waveform } & D(\mathrm{~cm}) & \bar{Q}\left(1 \mathrm{~min}^{-1}\right) & \text { Heart rate }\left(\mathrm{min}^{-1}\right) & \mu(\mathrm{Pa} \mathrm{s}) & R e & \text { Wo } \\ Q 1 \text { (rest) } & 1.7 & 0.8 & 72 & 0.004 & 264 & 12 \\ Q 2 \text { (exercise) } & 1.7 & 5.1 & 95 & 0.004 & 1700 & 13.8\end{array}$

TABLE 1. Values of flow parameters related to the flow rate waveforms recorded by Suh et al. (2011).

value one. Details of the two waveforms are given in table 1. In Suh et al. (2011) blood is considered to be a Newtonian fluid with a density of $1.06 \mathrm{~g} \mathrm{~cm}^{-3}$ and a viscosity of $0.004 \mathrm{~Pa}$ s. To convert the recorded volume flow rates to non-dimensional quantities, one also needs to know the diameters of the abdominal aorta at the locations of the recording. These values are not given in Suh et al. (2011). The values of the Reynolds number and Womersley number in table 1, for example $R e=256$ and $W o=12$ for waveform $Q 1$, are based on a vessel diameter of $1.7 \mathrm{~cm}$, which seems to be a typical value of the inlet diameter of an abdominal aneurysm. The same waveform $Q 1$ in a blood vessel with diameter $2.0 \mathrm{~cm}$ would correspond to $R e=250$ and $W o=15$. The coefficients $Q_{n}$ of a Fourier series representation of the waveforms, using 10 Fourier coefficients, are listed in table 2.

Given the fact that it is often easier to determine the blood flow rate waveform at a particular location than the values of the blood viscosity and the local arterial diameter, we have studied the characteristics of the pulsatile flow through the model aneurysm with the flow rate waveform $Q 1$ at the inlet, for three values of the Womersley number (10, 12 and 15) and for Reynolds numbers between 200 and 500. The characteristics of the flow with waveform $Q 2$ at inlet conditions that are mentioned in $\S 3$ are for $R e=1700$ and $W o=13.8$, as mentioned in table 1. However, it proved to be convenient for the study of the stability of the flow to set the Reynolds number to 500, while keeping 13.8 as the value of the Womersley number.

\subsection{Stability analysis}

To study the stability of the axisymmetric time-periodic flow to three-dimensional perturbations, the Navier-Stokes equations (2.2) are linearized around the axisymmetric time-periodic base flow. Let base flows be time periodic with period $T$ such that 


$\begin{array}{ccc}Q_{0} & 1.0 & 1.0 \\ Q_{1} & 0.4461-\mathrm{i} 0.9481 & 0.0203-\mathrm{i} 0.4735 \\ Q_{2} & -0.7231-\mathrm{i} 0.5638 & -0.1923-\mathrm{i} 0.0389 \\ Q_{3} & -0.3046+\mathrm{i} 0.3181 & -0.0392+\mathrm{i} 0.0068 \\ Q_{4} & 0.0042+\mathrm{i} 0.1447 & -0.0405+\mathrm{i} 0.0103 \\ Q_{5} & 0.0469+\mathrm{i} 0.1112 & -0.0054+\mathrm{i} 0.0170 \\ Q_{6} & 0.0780+\mathrm{i} 0.0169 & -0.0026+\mathrm{i} 0.0008 \\ Q_{7} & 0.0256-\mathrm{i} 0.0184 & -0.0009+\mathrm{i} 0.0023 \\ Q_{8} & 0.0192-\mathrm{i} 0.0104 & 0.0013+\mathrm{i} 0.0003 \\ Q_{9} & -0.0021-\mathrm{i} 0.0119 & -0.0002-\mathrm{i} 0.0022\end{array}$

(a)

(b)

TABLE 2. The coefficients $Q_{n}$ of the Fourier series representation of the waveforms shown in figure 3. (a) Rest: $Q 1,(b)$ exercise: $Q 2$.

$\boldsymbol{U}(r, z, t)=\boldsymbol{U}(r, z, t+T)$. Then, to this flow a three-dimensional perturbation velocity field $\boldsymbol{u}^{\prime}(r, z, \theta, t)$ is added to form the composite velocity field:

$$
\boldsymbol{u}(r, z, \theta, t)=\boldsymbol{U}(r, z, t)+\boldsymbol{u}^{\prime}(r, z, \theta, t) .
$$

Substitution of this expression into (2.2) and retaining terms that are linear in the perturbation velocities then yields the equations

$$
\begin{gathered}
\frac{\partial \boldsymbol{u}^{\prime}}{\partial t}+(\boldsymbol{U} \cdot \nabla) \boldsymbol{u}^{\prime}+\left(\boldsymbol{u}^{\prime} \cdot \nabla\right) \boldsymbol{U}=-\nabla p^{\prime}+\frac{1}{R e} \Delta \boldsymbol{u}^{\prime}, \\
\nabla \cdot \boldsymbol{u}^{\prime}=0 .
\end{gathered}
$$

The perturbations are taken to have the form

$$
\left[\begin{array}{l}
u_{r}^{\prime}(r, \theta, z, t) \\
u_{\theta}^{\prime}(r, \theta, z, t) \\
u_{z}^{\prime}(r, \theta, z, t) \\
p^{\prime}(r, \theta, z, t)
\end{array}\right]=\left[\begin{array}{l}
\hat{u}_{r}(r, z, t) \\
\hat{u}_{\theta}(r, z, t) \\
\hat{u}_{z}(r, z, t) \\
\hat{p}(r, z, t)
\end{array}\right] \exp \mathrm{i}(m \theta)+\text { c.c. }
$$

where $m$ is the azimuthal mode number. For the computations, a plane of symmetry for the perturbations is chosen by considering $\hat{u}_{r}(r, z, t), \hat{u}_{z}(r, z, t)$ and $\hat{p}(r, z, t)$ to be purely real and $\hat{u}_{\theta}(r, z, t)$ to be purely imaginary. This permits one to rewrite the above equations in terms of purely real variables, thereby reducing the computational cost.

The numerical analysis of the stability of the time-periodic flows is carried out by time-marching (2.5) for a suitable number of pulse cycles and monitoring the perturbation fields until they converge. Once the perturbations have converged, the evolution of the perturbation velocity fields at specific points in the computational domain is recorded to determine the Floquet multiplier. According to Floquet theory (Herbert 1988), the velocity and pressure perturbations grow or decay exponentially from period to period, thus

$$
\hat{u}(r, z, t+T)=\exp (\sigma T) \hat{u}(r, z, t),
$$

where $T$ is again the period of the pulsatile flow and $\sigma$ is the (complex) growth rate. The coefficient $\mu=\exp (\sigma T)$ is the so-called Floquet multiplier. The absolute value $|\mu|$ of the Floquet multiplier is computed as

$$
|\mu|=E_{k}(t+T) / E_{k}(t)
$$


with $E_{k}(t)$ defined as

$$
E_{k}(t)=\left\{\int_{\Omega}\left[u_{r}^{\prime}(t)^{2}+u_{\theta}^{\prime}(t)^{2}+u_{z}^{\prime}(t)^{2}\right] \mathrm{d} \Omega\right\}^{1 / 2} .
$$

For $|\mu|>1$ the flow is unstable, and for $|\mu|<1$ the flow is stable; a value $|\mu|=1$ represents neutral stability. Further, modes can be classified according to the value of the Floquet multiplier. A real bifurcation $(\mu=1)$ has the same period as the base flow, a subharmonic bifurcation $(\mu=-1)$ has a period of twice that of the base flow. Such a period-doubling bifurcation can be detected by investigating the perturbation field, which will alternate between opposite values from one period to the next. Complex-conjugate Floquet multipliers correspond to perturbation fields in the form of standing or travelling waves. A complex-conjugate bifurcation can be identified from the evolution of the absolute value of the Floquet multiplier. An oscillation around a mean value signifies that the Floquet multiplier is complex (Robichaux, Balachandar \& Vanka 1999; Sheard, Thompson \& Hourigan 2005).

\subsection{Numerical procedure}

The flow problem given by the equations and boundary conditions mentioned in $\S 2.2$ has been solved numerically by a finite-element method. The spatial discretization is a mixed finite-element formulation using P2-P1 Taylor-Hood elements: six-node quadratic triangular elements with quadratic interpolation for velocities $(\mathrm{P} 2)$ and threenode linear triangular elements for pressure (P1). The meshes, as well as the discrete matrices resulting from the variational formulation of the problem, are generated with the software FreeFem++ (http://www.freefem.org).

An inlet length of $10 D$ units and an outlet length of $20 D$ units have been used in the simulations. At the start of the calculation, the velocity field is considered to be given by the Womersley solution within the cylindrical domain corresponding to a straight vessel of constant cross-section, and a zero velocity field within the dilatation inflation. The flows are time-marched for a sufficient number of cycles for the mean flow to pass through the computational domain. Time traces of the velocities at various points within the domain are then checked at specific phases in the cycle, to confirm that the flows have converged to a time-periodic state. For the parameter conditions explored in the present study, the flow needed to be integrated in time for 10 cycles to attain periodicity. To these axisymmetric base flows, three-dimensional disturbances were added of the form given by (2.6), and the linearized Navier-Stokes equations (2.5) were time-marched for a sufficient number of pulse cycles to obtain the Floquet multipliers. The mesh and the numerical procedure are the same as used in the computation of the time-periodic basic flows.

Extensive validation tests have been performed, the details of which can be found in Gopalakrishnan (2014). Here, we merely present the results of validation tests that consisted of a computational study of pulsatile flow through model arterial stenoses, to verify our results against those presented by Griffith (2007). Table 3 presents a comparison between some of our results and those of Griffith (2007). The parameter $b$ is the stenosis degree, defined as

$$
b=1-(d / D)^{2},
$$

with $d$ the diameter of the vessel at the centre of the blockage; $L_{0}$ denotes the length of the vessel outlet in units of the vessel diameter and $A$ is the amplitude of the harmonic flow rate pulsation. 


$\begin{array}{cccccc}L_{0} & b & A & m & |\mu| & |\mu|_{g} \\ 30 & 0.50 & 1.25 & 1 & 1.0648 & - \\ 40 & 0.50 & 1.25 & 1 & 1.1741 & 1.1708 \\ 30 & 0.60 & 1.00 & 1 & 1.3495 & - \\ 50 & 0.60 & 1.00 & 1 & 1.3757 & 1.3761\end{array}$

TABLE 3. Comparison between the magnitude of the Floquet multiplier associated with mode $m=1$ of a perturbed pulsatile flow through a stenosis, as calculated by us, $|\mu|$, and by Griffith (2007), $|\mu|_{g}$.
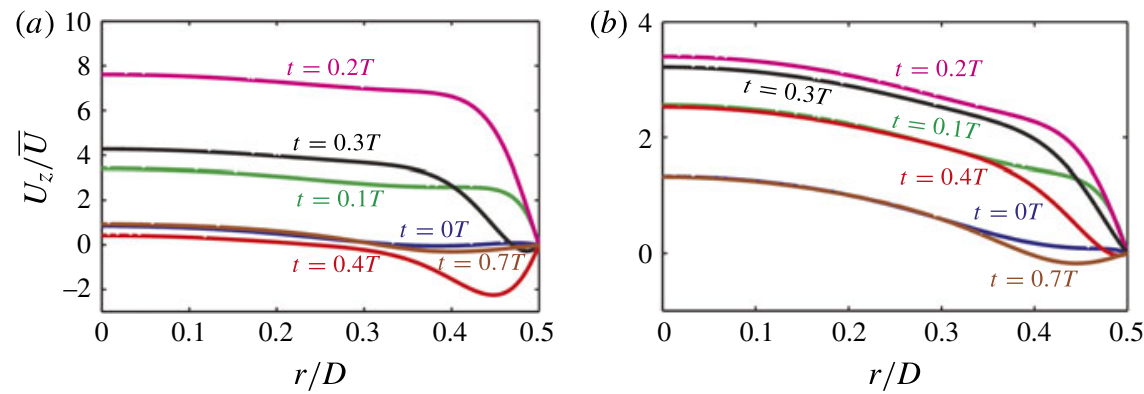

FIgURE 4. (Colour online) Evolution of the axial velocity profile in a straight vessel during one cardiac cycle: (a) $Q 1$ (rest); (b) $Q 2$ (exercise).

\section{The pulsatile flow}

\subsection{Flow through a vessel with constant cross-sectional area}

Before discussing flow through aneurysms, we look at some details of the imposed physiological waveforms.

As can be seen in figure $3(b)$, under resting conditions $(Q 1)$ the peak flow rate during systole $(t=0.18 T)$ goes up to five times the mean value. After peak systole the flow rate is reduced and even becomes negative during peak diastole $(t=0.44 T)$. At the end of the diastole, the flow rate becomes positive again and relaxes to zero during the resting period, before increasing once again at the beginning of the next cardiac cycle. Under exercise conditions $(Q 2)$ the flow rate remains positive during the entire cardiac cycle: already at the beginning of the cardiac cycle there is a significant flow rate and the flow does not reverse during diastole. Interestingly, the instantaneous velocity profiles during exercise are very similar to the instantaneous velocity profiles, at the same phase within the cycle, during rest; the difference essentially consists in the velocity distribution of a steady Poiseuille flow. This implies that the relative magnitude of the oscillatory component during rest is larger than during exercise conditions.

During the acceleration phase of the systole, the flow develops into a characteristic top-hat velocity profile, as can be seen in figure 4 . Thin boundary layers are observed which scale as $D / W o$. In line with what was said above, the velocity profiles observed under exercise conditions are more parabolic. The axial velocity also remains positive throughout the cardiac cycle except for a short duration during diastole, when negative velocities are found close to the wall.

The wall shear stress is generally considered as the primary fluid mechanical parameter with regard to the physiological response of the endothelial cells lining the 

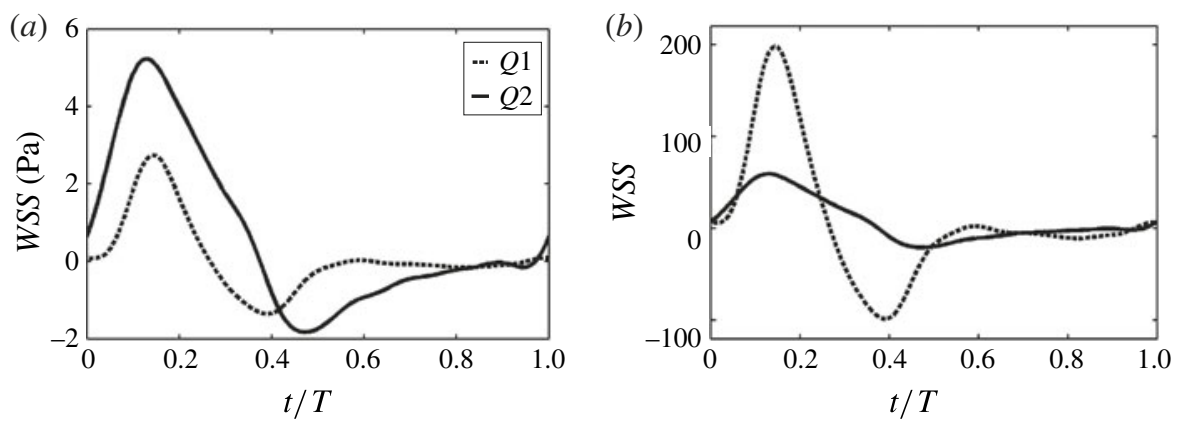

FIGURE 5. Evolution of the wall shear stress during one cardiac cycle, as calculated from the Womersley solution corresponding to the volume flow rate waveforms $Q 1$ (during rest) and $Q 2$ (during exercise). (a) Physical values based on the data given in table 1, (b) nondimensionalized by multiplying by $D / \mu \bar{U}$.

arterial wall $(\mathrm{Ku}$ 1997). The time variation of the wall shear stress can be determined from the velocity profiles described above by evaluating

$$
W S S(z, t)=-\left.\mu \frac{\partial U_{z}}{\partial r}\right|_{r=w a l l} .
$$

Here, $\mu$ represents the dynamic viscosity of blood. The convention is to assign a negative value to the function WSS in the case of reversed flow. Various quantities have been introduced by different authors to investigate the response of endothelial cells to wall shear stress variations. Examples are the cycle-averaged wall shear stress and the cycle-averaged magnitude of that stress, quantities that are defined as in Salsac et al. (2006),

$$
\overline{W S S}=\frac{1}{T} \int_{0}^{T} W S S \mathrm{~d} t, \quad \overline{|W S S|}=\frac{1}{T} \int_{0}^{T}|W S S| \mathrm{d} t,
$$

respectively. For later comparison, the temporal evolution of the wall shear stress under rest and exercise conditions in a healthy vessel is shown in figure 5; it simply follows the evolution of the flow rate. The peak wall shear stress under exercise conditions is almost twice that during rest conditions. The minimum value of the wall shear stress, however, does not change drastically under varying conditions. The time-averaged wall shear stress is roughly seven times higher during exercise than during rest, but the peak-to-mean ratio is almost four times higher under resting conditions than during exercise.

\subsection{Flow in abdominal aneurysms during rest}

\subsubsection{Phenomenology}

We consider a model aneurysm with $W=0.5$ and $H=0.5$ for a discussion of the typical flow features; the flow conditions are given by $R e=264$ and $W o=12$. Figure 6 shows the azimuthal vorticity component at various time instants during a pulse cycle. As can be seen in frame (iii), by the time the flow rate reaches peak systole a layer of (positive) vorticity has been formed. Subsequently, as the flow rate decreases, this layer of vorticity detaches from the wall and rolls up into a ring-like vortex structure 

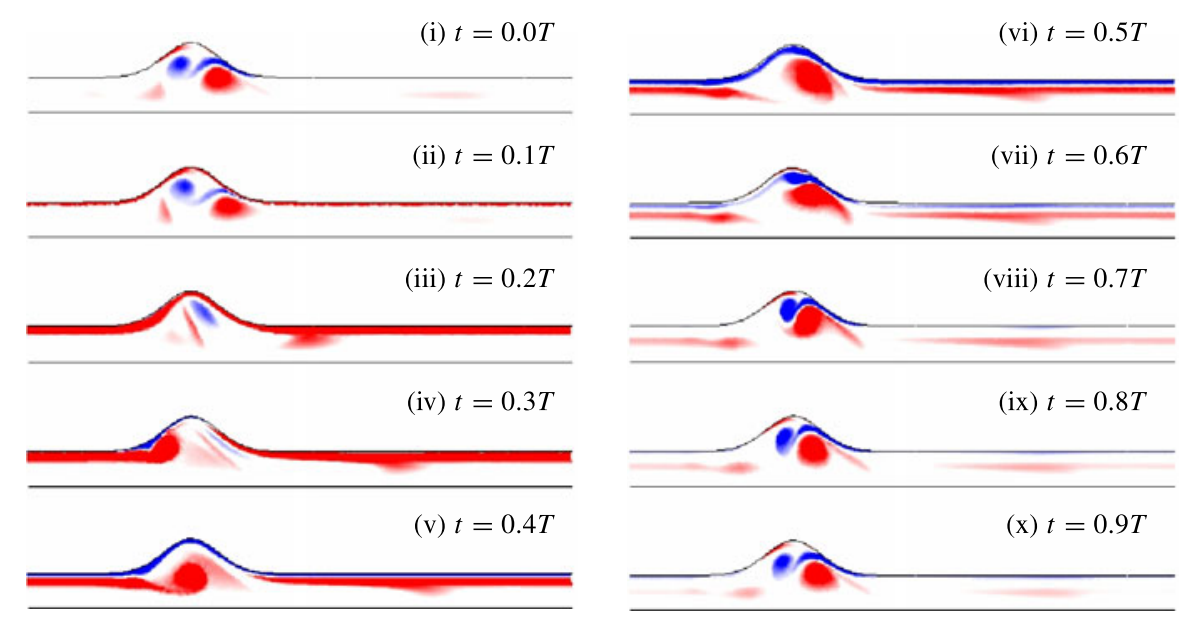

FIgURE 6. (Colour online) Evolution of the azimuthal vorticity distribution during one pulse cycle of the flow rate waveform $Q 1$ (rest conditions). The dimensionless vorticity values range from -20 (blue) to +20 (red). The axial range is $-3<z<7$ and the flow parameters and geometrical parameters have values $R e=264, W o=12, W=0.5, H=0.5$.

in the centre of the aneurysm, frames (iv) and (v), which moves towards the distal end of the dilatation, frames (vi)-(x). As a consequence, vorticity of opposite (negative) sign is produced at the wall, frames (v) and (vi). As this vorticity is torn off the wall it rolls up in a second ring-like vortex structure, frames (vii)-(x). The two ringlike vortex structures of opposite sign persist for a short period at the end of the cycle, frames (x) and (i), but as the flow rate increases again at the beginning of a new cycle, the primary (positively signed) vortex is washed away downstream, while the second (negatively signed) vortex is annihilated by the newly produced positively signed vorticity at the wall, frames (ii) and (iii). The process then repeats.

Differences between the wall shear stress distribution of a healthy artery and that of an artery with a local dilatation can be deduced by comparing figure 5 with figure 7 . In the latter figure, the axial variation of the cycle-averaged wall shear stress and the cycle-averaged magnitude of the wall shear are plotted. It will be seen that significant deviations from the values found for a healthy artery are limited to the central part of the dilatation, roughly in the range $-1<z<1$. The minimum and maximum values of the cycle-averaged wall shear stress magnitude in the dilated artery are $36 \%$ and $197 \%$, respectively, of the values found for the healthy artery. However, with regard to the cycle-averaged wall shear stress itself, it has a positive peak value for the inflated vessel that is 2.8 times the cycle-averaged shear stress in a healthy vessel, and, at another location, a negative peak with a level 4.8 times the mean shear stress in a healthy vessel. It should be noted that these oppositely signed peak values of the mean stress are located within a short distance of each other.

\subsubsection{Variations of the geometry}

The effect of varying the geometry, as represented by the dilatation ratio $D_{R}=1+2 H / D$, the ratio of the maximum diameter to the inlet diameter, is investigated by varying the non-dimensional bulge height $H$ for a fixed value of $W$. As a first example we take $W=0.5$. Figure 8 presents the vorticity distribution of the flow in the model aneuryrsm at various time instants during a cycle for the 


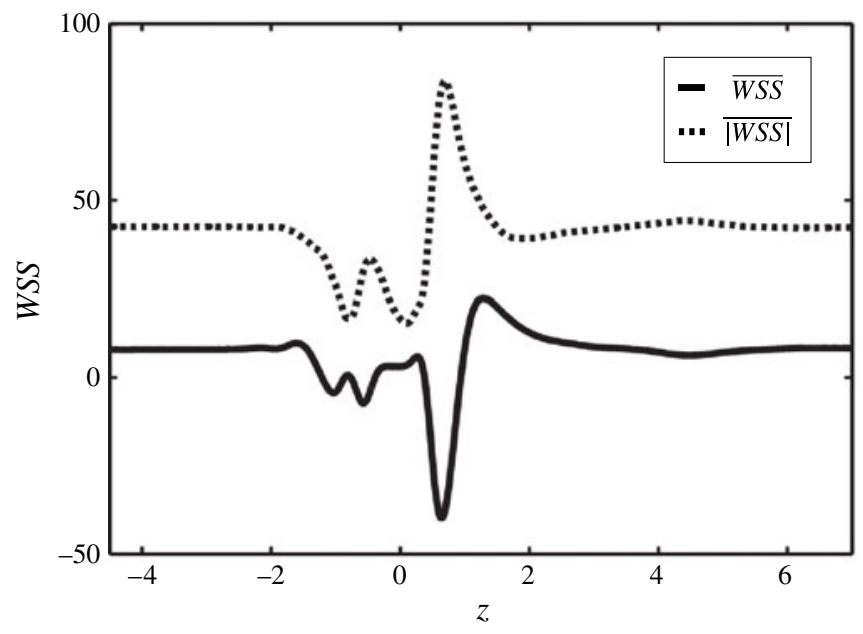

FIGURE 7. Axial variation of the cycle-averaged wall shear stress ( $\overline{W S S}$; solid line) and cycle-averaged magnitude of the wall shear stress ( $\overline{|W S S|}$; dotted line) in the model aneurysm, calculated for the flow rate waveform $Q 1$ (rest conditions) and with values of the flow parameters and geometrical parameters as in figure 6: $R e=264, W o=12, W=$ $0.5, H=0.5$.

values $H=0.3$ and $H=1.0$; the waveform and values of the Reynolds number and Womersley parameter are the same as in figure 6. It should be noted that the flow phenomenology described in the previous section for $H=0.5$ is also found for a value of the dilatation ratio as low as $D_{R}=1.6(H=0.3)$. However, in this shallow dilatation the primary vortex occupies a relatively larger volume and is more readily washed away and convected downstream. As a consequence, the secondary oppositely signed vortex structure occupies less volume and its formation is delayed. In a more developed dilatation, as characterized by $H=1.0$, the vortices remain trapped during a longer phase of the cycle and the secondary vortex persists throughout the whole cycle.

As a second example, we consider $W=1$, which is representative of a longer aneurysm than in the first example $(W=0.5)$, for the same value of $H$. Figure 9 presents the vorticity distribution in the dilatation, again for $H=0.3$ and $H=1.0$, and for the same waveform $Q 1$, Reynolds number and Womersley parameter as in figure 6. The differences are remarkable: the layer of vorticity at the wall remains attached for a much larger part of the cycle, and, as a result, the primary ring-like vortex structure forms much later during the deceleration phase after peak systole and remains much weaker. The interaction with the wall is so weak that the primary vortex does not move towards the distal wall and no secondary oppositely signed vortex structure is formed during diastole.

The distributions of the cycle-averaged wall shear stress associated with the examples just given are summarized in figure 10, where results for $H=0.7$ have also been added. A first conclusion from these plots is that the peak value of the cycle-averaged wall shear stress does not seem to be a significant quantity to monitor to decide on the growth of an aneurysm, as it varies very little with changes of $H$. A similar conclusion could be drawn regarding the distribution itself, since that also remains qualitatively similar as $H$ increases while $W$ is kept fixed. It should be noted, 
(a)

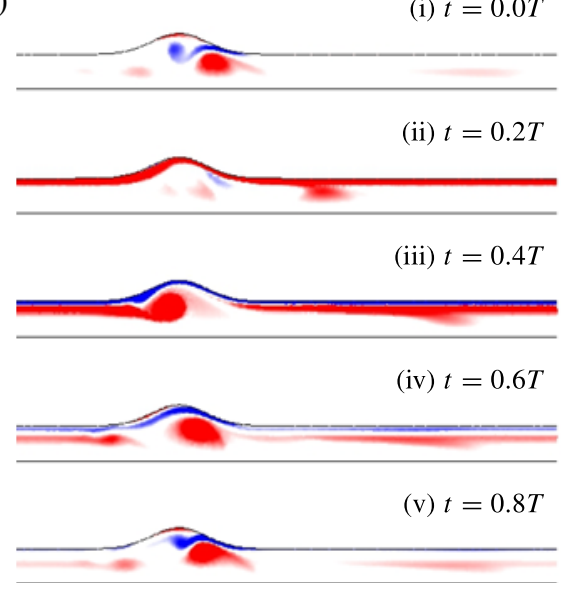

(b)
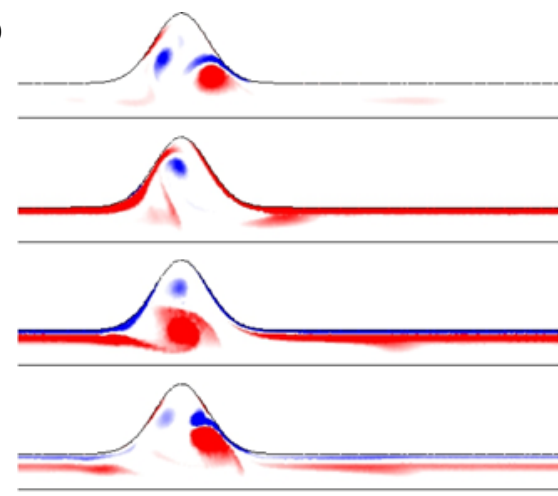

$\cdot$

FIGURE 8. (Colour online) Evolution of the azimuthal vorticity distribution during one pulse cycle of the flow rate waveform $Q 1$ (rest conditions). The dimensionless vorticity values range from -20 (blue) to +20 (red). The axial range is $-3<z<7$ and the flow parameters and geometrical parameters have values $R e=264, W o=12, W=0.5 ; H=0.3$ in $(a)$ and $H=1.0$ in $(b)$.

(a)

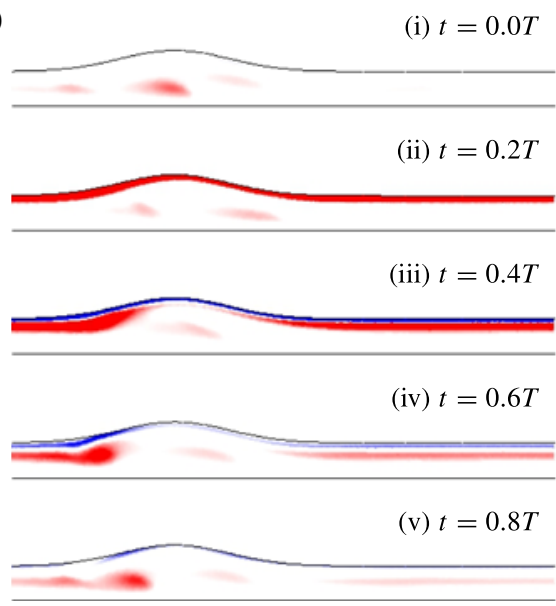

(b)

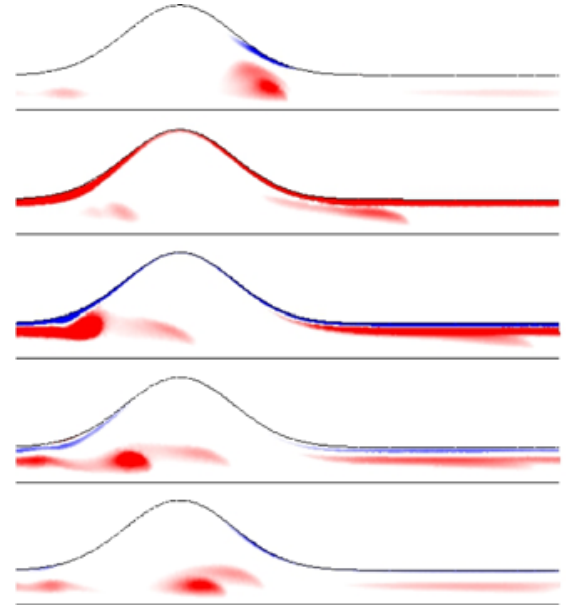

FIGURE 9. (Colour online) Evolution of the azimuthal vorticity distribution during one pulse cycle of the flow rate waveform $Q 1$ (rest conditions). The dimensionless vorticity values range from -20 (blue) to +20 (red). The axial range is $-3<z<7$. The flow parameters and geometrical parameters have the same values as in figure $8: \operatorname{Re}=$ 264, $W o=12, H=0.3$ in $(a)$ and $H=1.0$ in $(b)$, but here $W=1.0$.

however, that much stronger spatial gradients of the cycle-averaged wall shear stress are found for $W=0.5$ than for $W=1.0$.

\subsubsection{Variations of the flow parameters}

In this section we consider the effects of varying the Reynolds number and Womersley number. The geometry is characterized by $W=0.5$ and $H=0.5$, values 

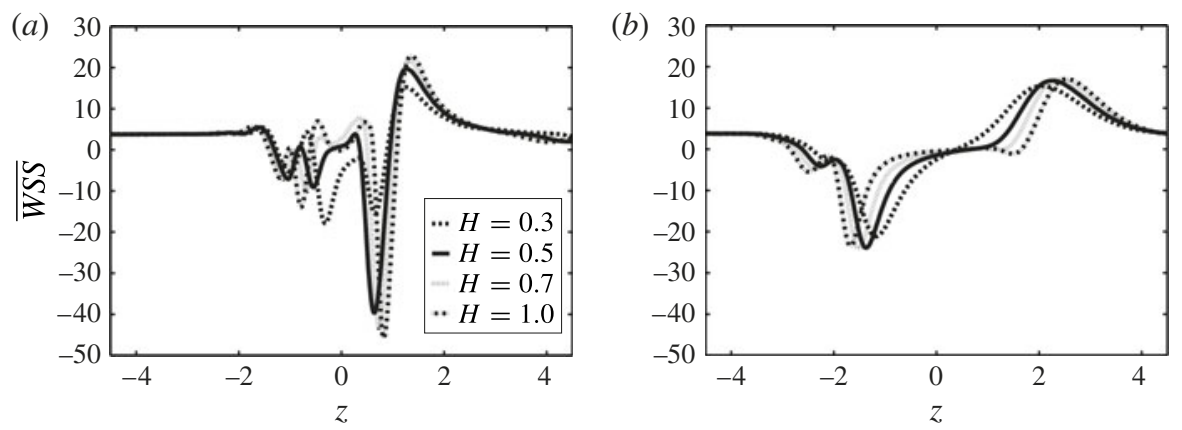

FIGURE 10. Axial variation of the cycle-averaged wall shear stress $(\overline{W S S})$ in the model aneurysm, as calculated for the flow rate waveform $Q 1$ (rest conditions) and with values of the flow parameters as in figure 7: $R e=264, W o=12$, but here $W=0.5$ in $(a)$ and $W=1.0$ in $(b)$, while the values of $H$ vary between 0.3 and 1.0.

for which the vorticity distribution and cycle-averaged wall shear stress have already been shown in figures 6 and 7, for $R e=264$ and $W o=12$.

In the first example the value of the Reynolds number is kept fixed at 264, but the Womersley number has the values 10 and 15. Figure 11 presents the vorticity distribution at various instants during the pulse cycle. It shows that the flow characteristics for $W o=12$ are very different from those for $W o=15$. It should be noted, however, that with all other parameters kept fixed, an increase in the Womersley number corresponds to an increase of the square of the frequency of pulsation $\left(\omega \propto W o^{2}\right)$, so that a change from $W o=10$ to $W o=15$ means that the pulse frequency increases by more than a factor of two. The phenomenology for $W o=10$ is similar to that for $W o=12$, with a layer of vorticity that separates from the wall and rolls up to form a ring-like vortex structure. For $W o=15$ the wall shear layer remains attached for a longer part of the cycle and the rolling up of the vorticity is less prominent. Moreover, the vorticity in the structure that is formed is weaker and the structure does not move towards the distal wall of the inflation; as a result also no oppositely signed vortex structure is formed.

In the second example, the values of the Reynolds number are 200 and 500, while the value of the Womersley number is kept at 12 . Figure 12 indicates that the principal effect of increasing the Reynolds number is that the magnitude of the vorticity is larger in the primary vortex structure that forms during the deceleration phase after peak systole. Flow separation occurs at an earlier moment in the cycle, the vortex impinges at the distal wall and induces the formation of various vortices of alternating sign everywhere in the dilatation.

These observations are reflected in figure 13, where the corresponding cycleaveraged wall shear stress distributions are presented. Figure 13(a,b) show that the peak values of the cycle-averaged wall shear stress are reduced as the Womersley number is increased, and figure $13(c, d)$ show that an increase of the Reynolds number results in stronger spatial variations in the cycle-averaged stress.

\subsection{Flow in abdominal aneurysms during exercise}

To follow up on the remark just made, this section presents a few results for the waveform $Q^{2}$ (figure 3). The geometry is characterized by $H=0.5$ and $W=0.5$, and 
(a)

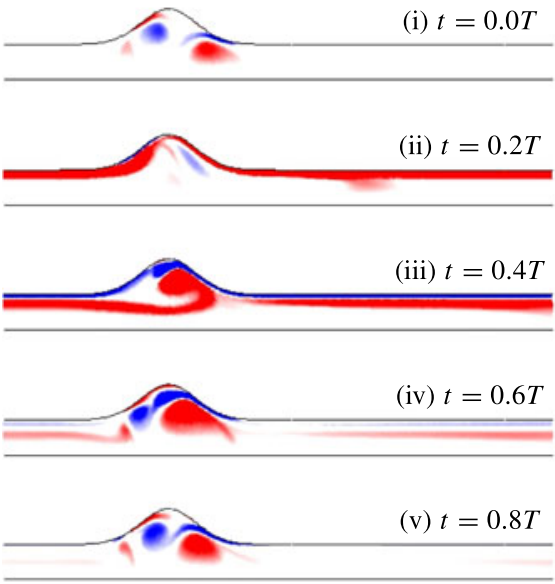

(b)
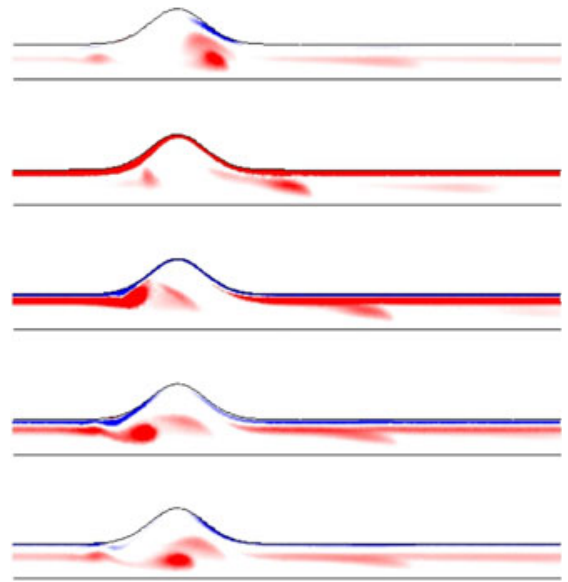

FIGURE 11. (Colour online) Evolution of the azimuthal vorticity distribution during one pulse cycle of the flow rate waveform $Q 1$ (rest conditions). The dimensionless vorticity values range from -20 (blue) to +20 (red). The axial range is $-3<z<7$. The flow parameters and geometrical parameters have the same values as in figure 6: $R e=264$, $W=0.5, H=0.5$, but here $W o=10$ in $(a)$ and $W o=15$ in $(b)$.

(a)

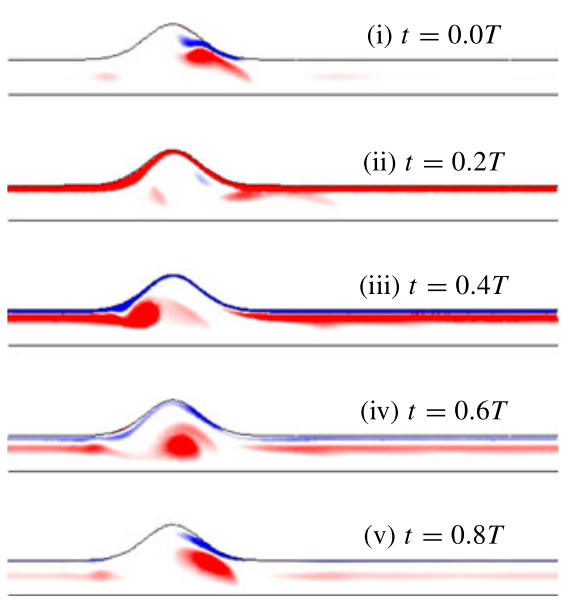

(b)
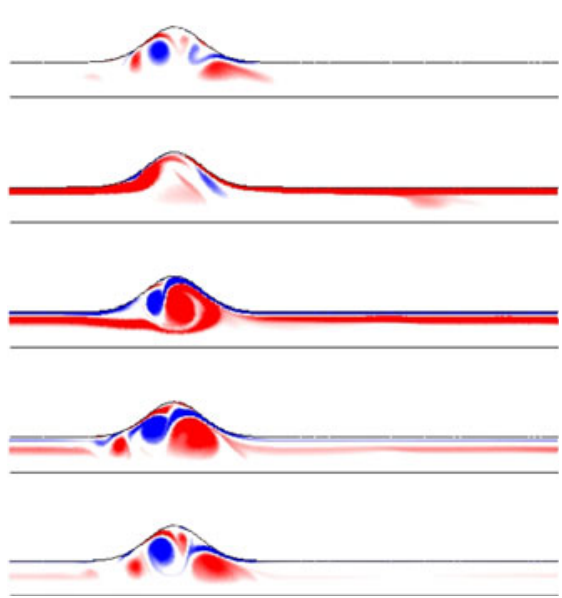

FIGURE 12. (Colour online) Evolution of the azimuthal vorticity distribution during one pulse cycle of the flow rate waveform $Q 1$ (rest conditions). The dimensionless vorticity values range from -20 (blue) to +20 (red). The axial range is $-3<z<7$. The flow parameters and geometrical parameters have the same values as in figure 6: $W o=12$, $W=0.5, H=0.5$, but here $R e=200$ in $(a)$ and $R e=500$ in $(b)$.

the flow by $W o=13.8$ and $R e=1700$. Figure 14 shows the vorticity distribution during various instants in a pulse cycle. At this high Reynolds number an intense separated shear layer is formed at the proximal end of the aneurysm during flow deceleration after peak systole. This shear layer rolls up and impinges at the distal end, where subsequently an oppositely signed secondary vortex structure is formed. This is similar to what was observed earlier for the waveform $Q 1$, typical of rest conditions, but in 

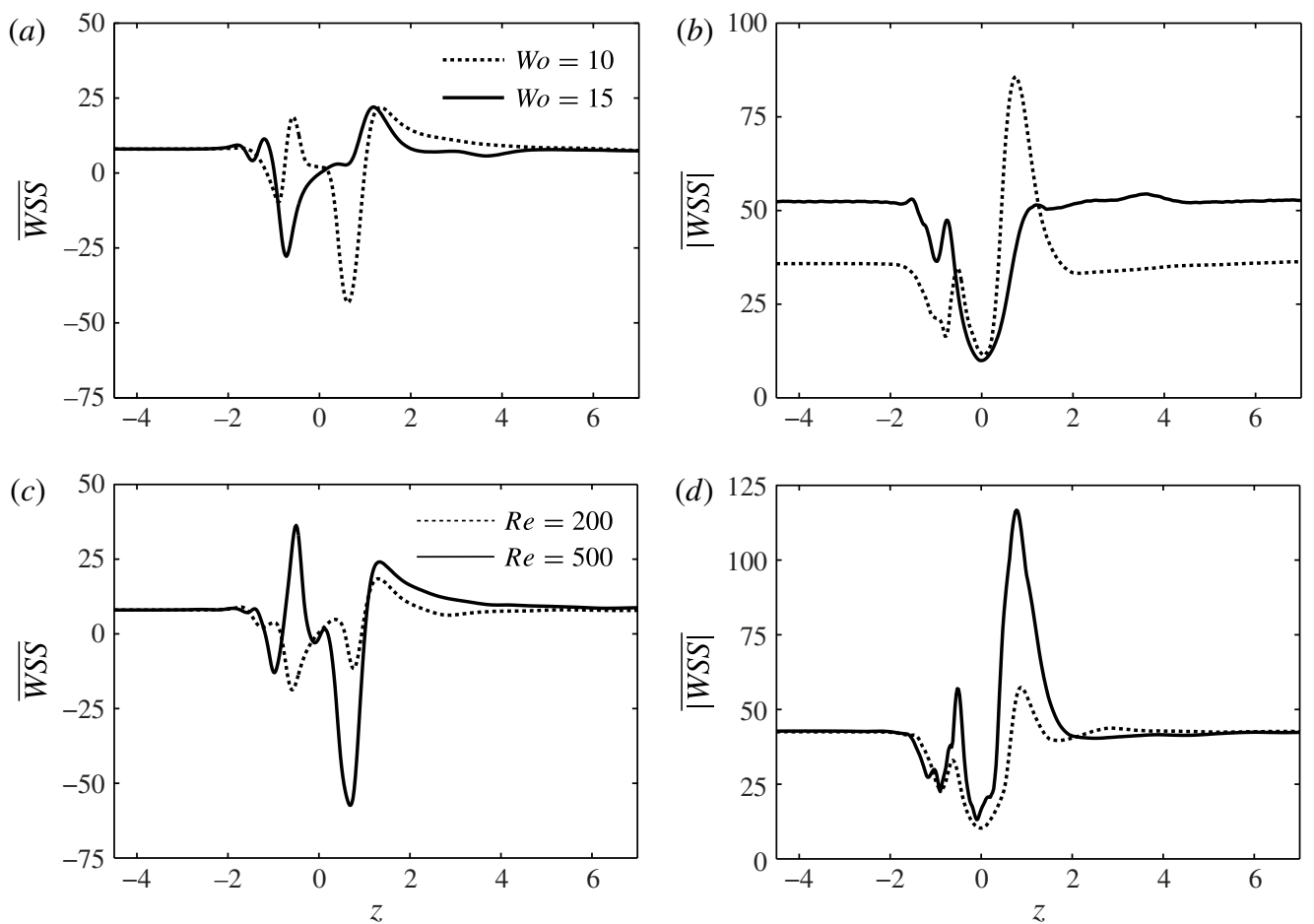

FIgURE 13. (a,c) Axial variation of the cycle-averaged wall shear stress $(\overline{W S S})$ and $(b, d)$ cycle-averaged magnitude of the wall shear stress $(\overline{|W S S|})$ in the model aneurysm, as calculated for the flow rate waveform $Q 1$ (rest conditions) and with values of the geometrical parameters as in figure 7: $W=0.5, H=0.5$. The flow parameters have the values $(a, b) \operatorname{Re}=264$, Wo $=10$ or $W o=15 ;(c, d) \operatorname{Re}=200$ or $\operatorname{Re}=500$, Wo $=12$.

the present case, because the vortices are much stronger, a tertiary vortex with the same sign as the primary vortex is induced inside the dilatation during the diastolic phase. This can be seen clearly in frame (vi), where the (negatively signed) secondary vortex pulls (positively signed) vorticity out of the shear layer, which then rolls up to become a positively signed tertiary vortex. The secondary and tertiary vortices then move together and collide with the proximal wall of the dilatation. The vortices are found to persist during most of the pulse cycle, but gradually weaken by diffusive effects.

Similar flows are observed for other values of $H$ and $W$. The features of the flows are reflected in the cycle-averaged wall shear stress distributions presented in figure 15. The height $H$ appears to have a relatively weak influence, except for with regard to the minimum value of the averaged wall shear stress. As the aneurysms grow in size, as represented by larger values of $H$, the secondary vorticity structure persists longer inside the dilatation; this appears to correlate with higher absolute values of the wall shear stress. Variations of the length of aneurysms, as represented by variations in the width $W$, correspond to shifts in the locations of the maximum and minimum values of the cycle-averaged wall shear stress.

Perhaps the most significant result from these observations is that at elevated Reynolds numbers the flow fields are highly complex, with vortices of alternating 
(i) $t=0.0 T$

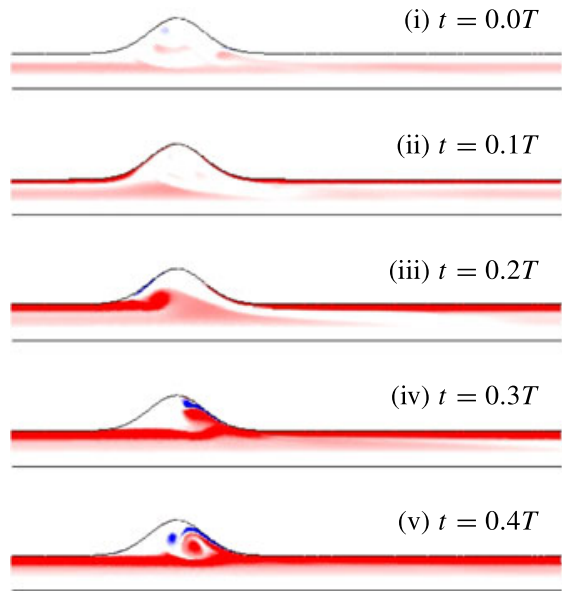

(vi) $t=0.5 T$

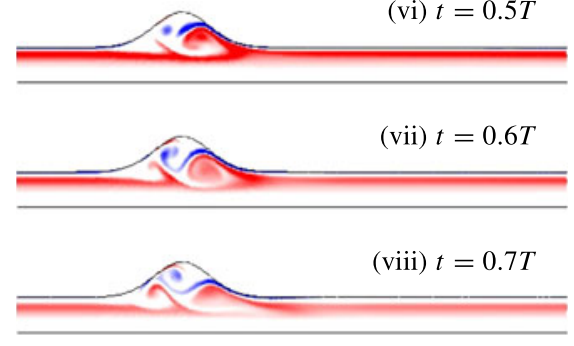

(ix) $t=0.8 T$

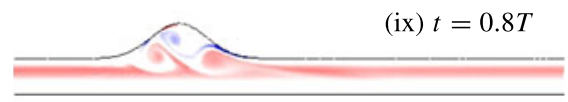

(x) $t=0.9 T$

FIgURE 14. (Colour online) Evolution of the azimuthal vorticity distribution during one pulse cycle of the flow rate waveform $Q 2$ (exercise conditions). The dimensionless vorticity values range from -20 (blue) to +20 (red). The axial range is $-3<z<7$. The flow parameters and geometrical parameters have values $R e=1700, W o=13.8, W=0.5$, $H=0.5$.

sign present throughout the aneurysm. As mentioned in $\S 1$, we believe that if such flow conditions persist, this may have a detrimental effect on the vessel wall.

\section{Stability characteristics}

\subsection{Flow in abdominal aneurysms during rest}

\subsubsection{Variations of the flow parameters}

We begin this discussion of the stability of pulsatile flows in a model aneurysm by looking at the flow associated with the pulse waveform $Q 1$. The parameters have values $H=0.5, W=0.5, W o=15$, while three values of the Reynolds number have been investigated: $R e=200, R e=250$ and $R e=300$. The results are summarized in figure 16. The pulsatile flow is stable at $R e=200$ and $R e=250$, but at $R e=300$ it is unstable to perturbations with mode numbers $m=2, m=3$ and $m=4$. The most unstable mode is that with $m=3$. In figure 16 the modes that are classified as subharmonic (period-doubling) are indicated by filled circles. The unstable modes can be seen to arise from two different sets of eigenmodes: the subharmonic modes dominate at small mode numbers, the harmonic modes at higher values of the mode number. It may be noted that something similar was observed for stenotic flows (Sherwin \& Blackburn 2005; Griffith et al. 2009), where the mode $m=1$ was found to correspond to a period-doubling bifurcation.

The vorticity distributions of the perturbations associated with modes $m=1$ and $m=3$ at various instants in a pulse cycle are shown in figure 17 for $R e=300$. They are arranged in bands and, although the modes are dominant inside the dilatation, they extend far downstream. This is especially the case for mode $m=1$, but appears to be a characteristic feature of all subharmonic modes. Such structures of the vorticity perturbations are very different from those that have been found for steady flows through similarly shaped model aneurysms (Gopalakrishnan, Pier \& Biesheuvel 2014); in that case these vorticity perturbations are confined to the dilatation. 

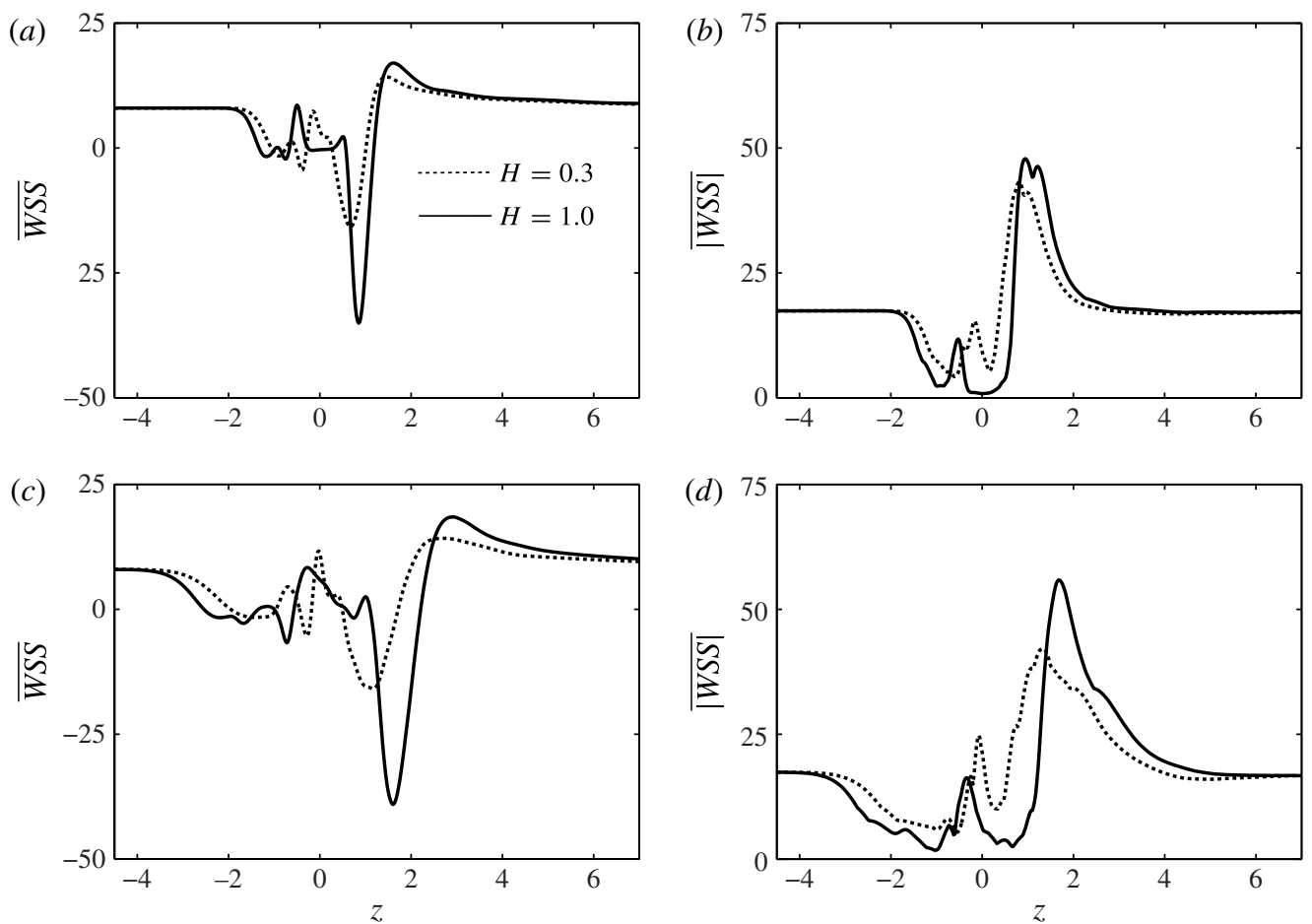

FIGURE 15. $(a, c)$ Axial variation of the cycle-averaged wall shear stress $(\overline{W S S})$ and $(b, d)$ cycle-averaged magnitude of the wall shear stress $(\overline{|W S S|})$ in the model aneurysm, as calculated for the flow rate waveform $Q 2$ (exercise conditions). The values of the flow parameters and geometrical parameters are $R e=1700, W o=13.8, H=0.3$ or $H=1.0$, $W=0.5(a, b)$ and $W=1.0(c, d)$.

These observations imply that the presence of an abdominal aortic aneurysm may create, for certain flow conditions, disturbed flows which extend far downstream into healthy sections of the arterial system. The result is that healthy vessel walls become exposed to sustained abnormal flow velocity conditions, which eventually may damage these vessel walls. This, in turn, may lead to atherosclerosis (Barakat 2013), or to the formation of a secondary aortic aneurysm. This might explain why about one-fifth of large abdominal aortic aneurysms are accompanied by aneurysms of the common iliac arteries.

Figure 18 summarizes the results of a stability analysis of the pulsatile flow at the same values of the parameters as in figure 16, except that now $W o=10(a)$ and $W o=$ $12(b)$. The most significant observation seems to be that as the Womersley number is reduced, the flows become unstable at lower values of the Reynolds number. As already mentioned, the flows at $W o=10$ and $W o=12$ are rather different from that at $W o=15$. The most unstable mode appears to be the mode $m=2$, which in the majority of cases can be classified as period-doubling. The absolute values $|\mu|$ of the Floquet multipliers are much higher for $W o=10$ and $W o=12$ than for $W o=15$. It should be noted, however, that the time periods $T \propto W^{-2}$ are longer too at Womersley numbers 10 and 12, in comparison with 15. Since the temporal growth rate $\sigma(=\ln \mu / T)$ is inversely proportional to the time period $T$, the growth rates at $W o=10,12$ are not 


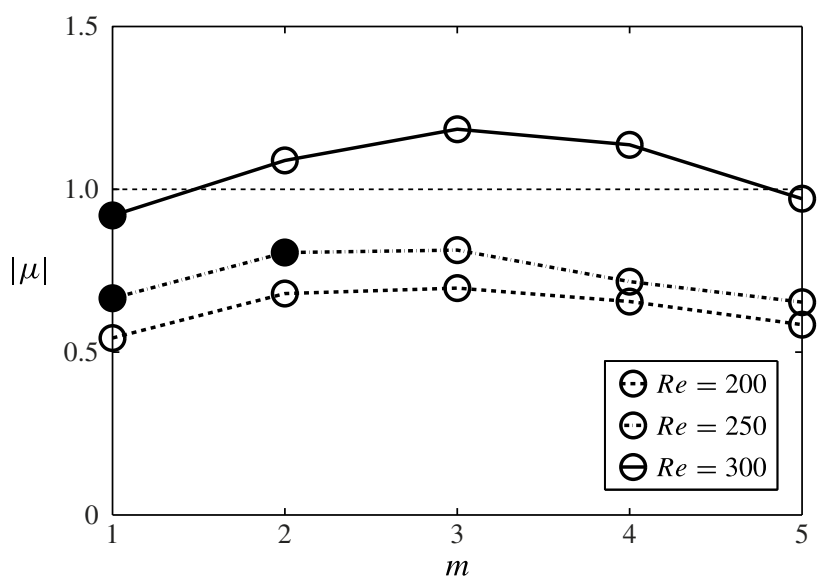

FIGURE 16. Variation of the absolute value of the Floquet multiplier $\mu$ with azimuthal mode number $m$ for pulsatile flow through a model aneurysm, for three different values of the Reynolds number. The flow rate waveform is $Q 1$ (rest) and the other parameters have the values $W o=15, H=0.5$ and $W=0.5$. Subharmonic modes are indicated by filled circles. The dotted line for $|\mu|=1$ is the stability boundary.

(a)
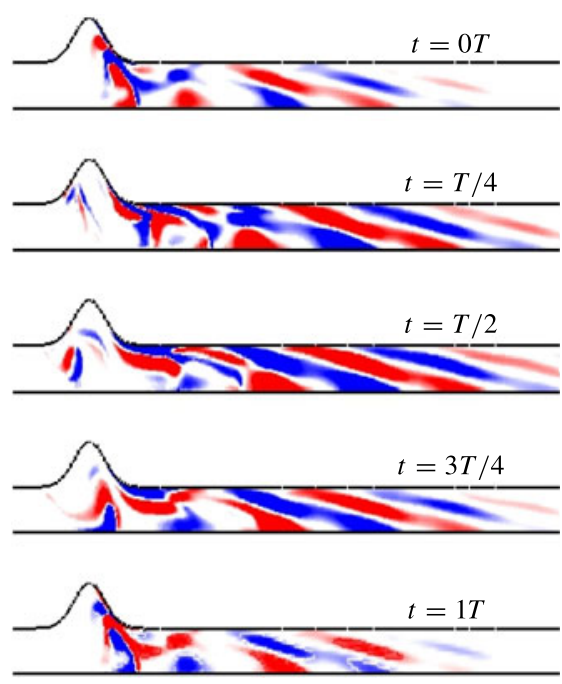

(b)
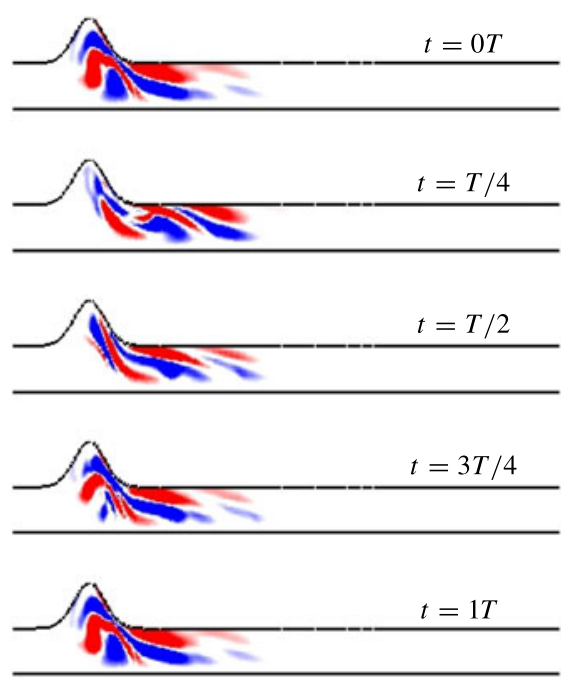

FIGURE 17. (Colour online) Evolution during one pulse cycle of the azimuthal vorticity distributions of the perturbed flows associated with the azimuthal modes $m=1(a)$ and $m=3(b)$. The basic flow is that of the pulse waveform $Q 1$ (rest) and the values of the flow parameters and geometrical parameters are $R e=300, W o=15, H=0.5, W=0.5$.

significantly higher than at $W o=15$, as the amplification of the perturbations takes place over a longer time.

\subsubsection{Response to harmonic forcing}

The presence of vorticity perturbations downstream of the aneurysm, examples of which are shown in figure 17, suggests that an abdominal aortic aneurysm can be 

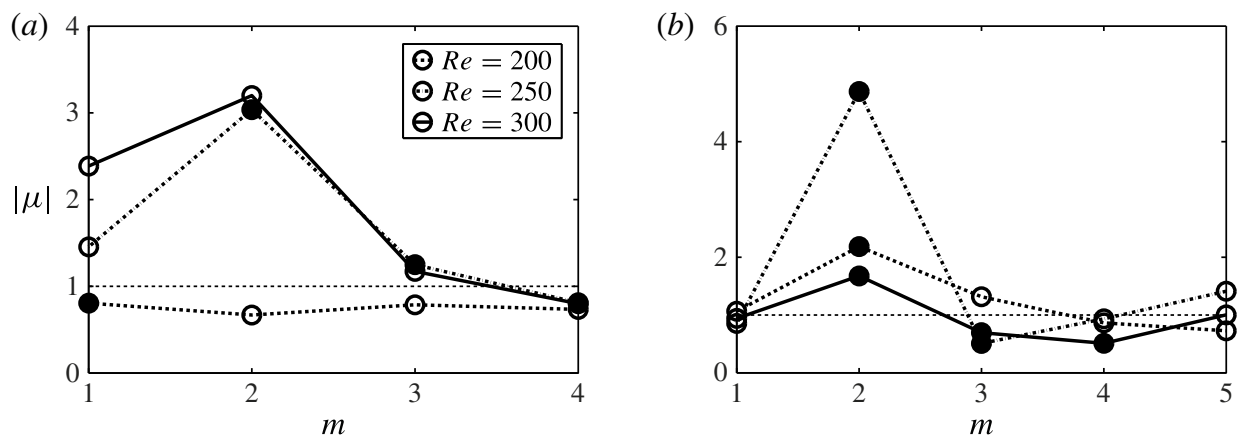

FIGURE 18. Variation of the absolute value of the Floquet multiplier $\mu$ with azimuthal mode number $m$ for pulsatile flow through a model aneurysm, for three different values of the Reynolds number. The flow rate waveform is $Q 1$ (rest) and the geometrical parameters have the same values as in figure $16, H=0.5$ and $W=0.5$, but here $W o=12(a)$ and $W o=10(b)$. Subharmonic modes are indicated by filled circles and the dotted line for $|\mu|=1$ is the stability boundary.

viewed as acting as a wavemaker which forces the flow in the vessels downstream. To verify this, we consider a straight circular vessel of sufficient length, and apply a harmonic forcing and observe the linear response. The unperturbed flow in the circular vessel is the Womersley solution. The external forcing is modelled as a body force $\boldsymbol{f}(\boldsymbol{x}, t)$ added to the linearized Navier-Stokes equations (2.5),

$$
\begin{aligned}
\frac{\partial \boldsymbol{u}^{\prime}}{\partial t}+(\boldsymbol{U} \cdot \nabla) \boldsymbol{u}^{\prime}+\left(\boldsymbol{u}^{\prime} \cdot \nabla\right) \boldsymbol{U} & =-\nabla p^{\prime}+\frac{1}{R e} \Delta \boldsymbol{u}^{\prime}+\boldsymbol{f}(\boldsymbol{x}, t), \\
\nabla \cdot \boldsymbol{u}^{\prime} & =0,
\end{aligned}
$$

where

$$
\boldsymbol{f}(\boldsymbol{x}, t)=\psi(\boldsymbol{x}) \exp \left(-\mathrm{i} \sigma_{i} t\right) .
$$

Here, the weight function $\psi(\boldsymbol{x})$ is chosen such that the forcing is restricted to a small region of space. The forcing frequency $\sigma_{i}$ is taken as the imaginary part of the complex growth rate obtained from the linear stability analysis of the flow through the model aneurysm.

Figure $19(b, d)$ shows the response of pulsatile flow in a straight circular vessel to harmonic forcing in comparison with the associated aneurysm flow (figure 19a,c) at two different flow conditions: $(a, b) R e=200, W o=15, m=1$ and $(c, d) R e=300$, $W o=15, m=3(H=0.5, W=0.5)$. The region of application of the forcing is the black circular spot. The perturbed flow downstream of the aneurysm (figure 19a,c) compares very well with the forced flow in $(b, d)$.

\subsubsection{Variations of the geometry}

Figure 20 summarizes the results of a stability analysis of the pulsatile flow at $R e=$ 264 and $W o=12$, for various values of $H$, while the value of $W$ is kept fixed at $W=0.5$ in $(a)$ and at $W=1.0$ in $(b)$. The radial extent of the dilatation, as represented by the value of $H$, is found to be of significance for the stability of the flow. For $W=0.5$, the flow is unstable for $H \geqslant 0.4$, the dominant mode in all cases being a subharmonic mode of azimuthal mode number 2. An additional calculation at $H=0.4$ was carried out because of the substantial variation that was observed of the values of 

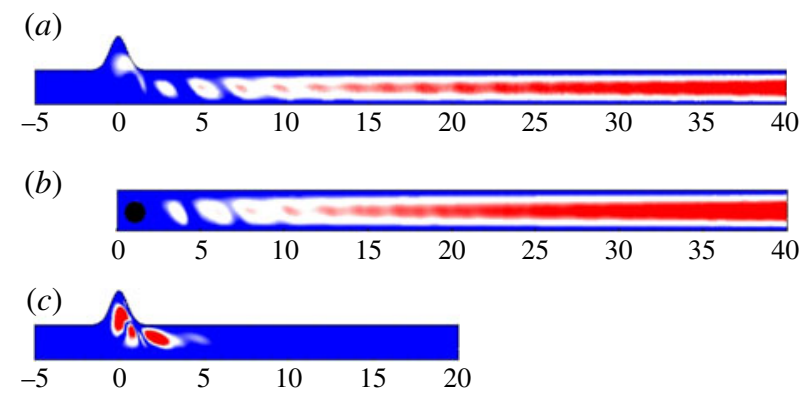

(d)

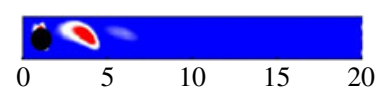

FIGURE 19. (Colour online) (a,c) Energy distribution of the axial velocity component of the leading critical mode for pulsatile flow through a model aneurysm; $(b, d)$ energy distribution of the axial velocity component of the perturbation flow set up by a harmonic forcing at the same frequency applied in the domain shown by a black circle of the pulsatile flow through a straight vessel at the same values of the Reynolds number and Womersley number. Parameter settings: $W=0.5, H=0.5,(a, b) \operatorname{Re}=200, W o=15, m=1$, $(c, d) \operatorname{Re}=300$, Wo $=15, m=3$.
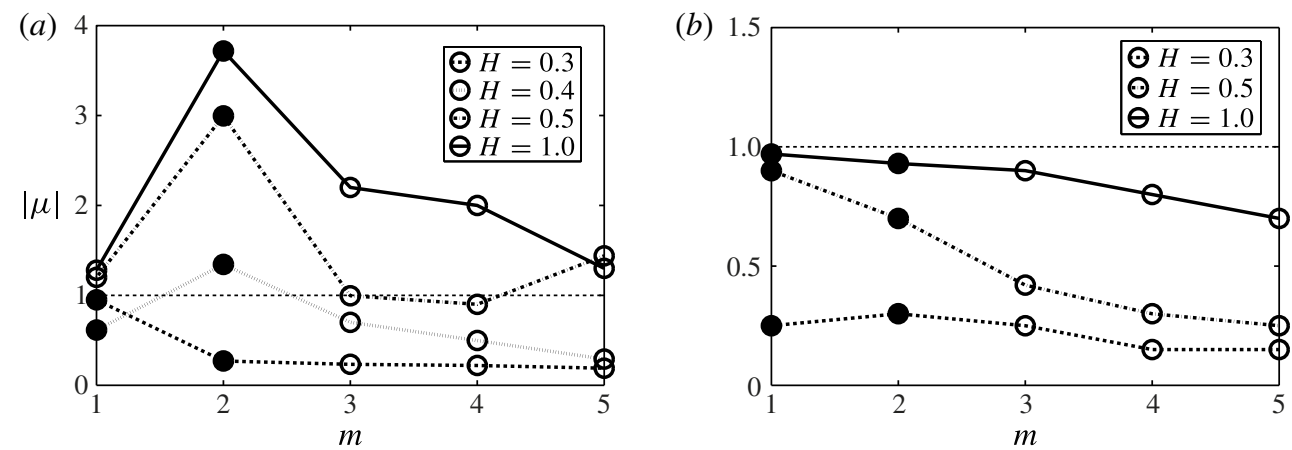

FIGURE 20. Variation of the absolute value of the Floquet multiplier $\mu$ with azimuthal mode number $m$ for pulsatile flow through a model aneurysm, for various values of the depth of the aneurysm, as represented by $H$. The flow rate waveform is $Q 1$ (rest) and the other parameters have the values $R e=264$, Wo $=12, W=0.5(a)$ and $W=1.0(b)$. Subharmonic modes are indicated by filled circles and the dotted line for $|\mu|=1$ is the stability boundary.

the Floquet multipliers as $H$ was changed from 0.3 to 0.5 . As expected, and confirmed in figure $20(b)$, the flow in a long aneurysm, as represented by the value of $W$, is less prone to becoming unstable than that in a short aneurysm.

The azimuthal vorticity distributions associated with the least stable modes of the flows in figure $20(a)$ are presented in figure 21. It is remarkable that already at the small value of $H=0.3$, the dominant mode extends downstream of the dilatation. Similar features are observed for $W=1$ in figure 22. We have already mentioned the observation that an aneurysm may initiate disturbed flow conditions in healthy segments of the arteries downstream of the aneurysm; the results shown in figures 21 and 22 now suggest that this will already occur in the incipient stages of an aneurysm. 


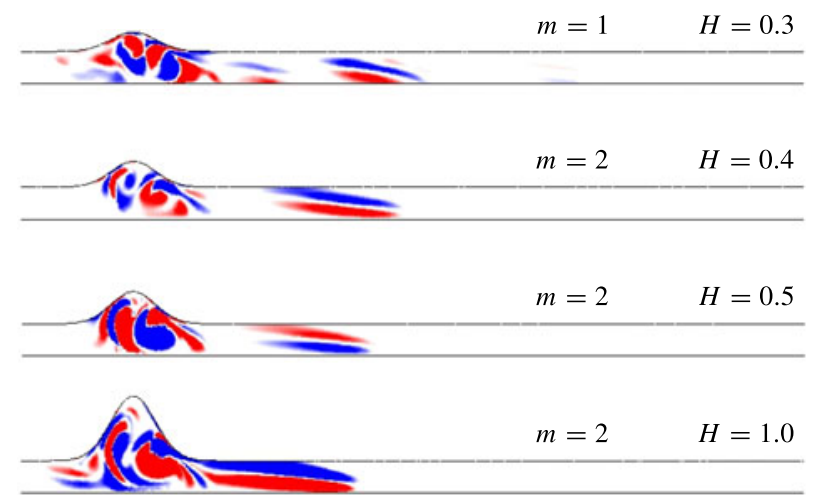

FIGURE 21. (Colour online) Azimuthal vorticity distributions of the perturbed flows associated with the least stable mode $m$ for pulsatile flow through a model aneurysm. The axial range shown is $-1.5 D \leqslant z \leqslant 7 D$ and the flow parameters and geometrical parameters have the values $R e=264, W o=12, W=0.5$ and from top to bottom $H=0.3,0.5,1.0$. The flow rate waveform is $Q 1$ (rest).

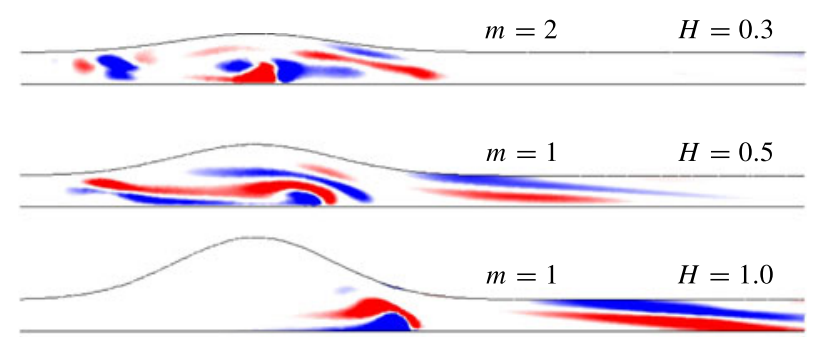

FIGURE 22. (Colour online) Azimuthal vorticity distributions of the perturbed flows associated with the least stable mode $m$ for pulsatile flow through a model aneurysm. The axial range shown is $-1.5 D \leqslant z \leqslant 10 D$ and the flow parameters and geometrical parameters have the values $R e=264, W o=12, W=1.0$ and from top to bottom $H=0.3,0.4,0.5,1.0$. The flow rate waveform is $Q 1$ (rest).

Clearly, this study is not conclusive, since the presence of the iliac artery bifurcation downstream of an abdominal aortic aneurysm can be expected to interact with the flow inside the aneurysm. Yet, what it does show is that the iliac bifurcation and segment of the common iliac arteries needs to be included in the computational domain when carrying out realistic, patient-specific simulations of flows in abdominal aortic aneurysms.

\subsection{Flow in abdominal aneurysms during exercise}

To conclude, we briefly consider the stability of pulsatile flow in the model aneurysm under exercise conditions. The waveform $Q 2$, recorded by Suh et al. (2011) and shown in figure $3(b)$, corresponds to a Reynolds number of 1700 and a Womersley number of 13.8, if the vessel diameter at the location of the recording is $1.7 \mathrm{~cm}$. However, to simplify the computations and to focus on the influence of the waveform, we have chosen the lower value $R e=500$ and kept the value $W o=13.8$. Nevertheless, we believe that the results provide a qualitative picture of the flow perturbations 


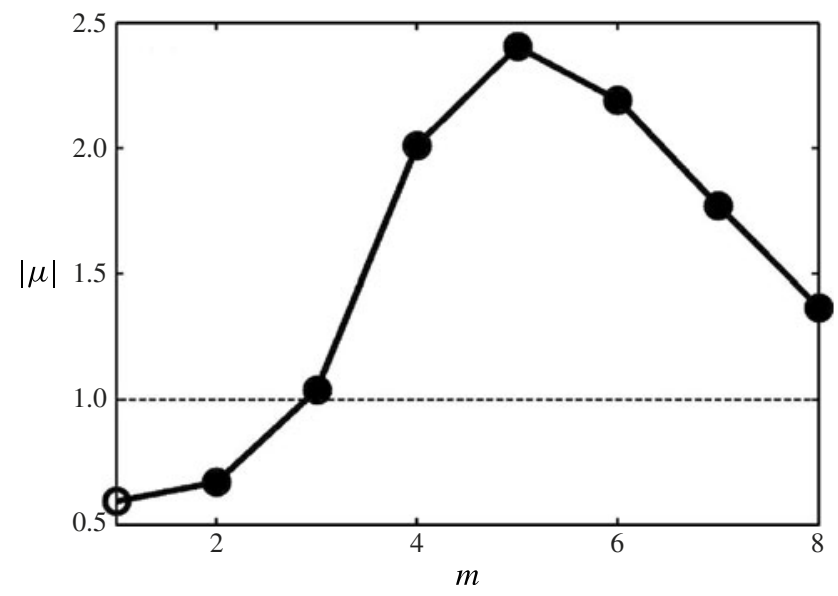

FIGURE 23. Variation of the absolute value of the Floquet multiplier $\mu$ with the azimuthal mode number $m$ for pulsatile flow through a model aneurysm. The flow parameters and geometrical parameters have the values $R e=500, W o=13.8, W=0.5, H=0.5$. The Womersley number corresponds to the flow rate waveform $Q 2$ (exercise), but the Reynolds number is reduced from 1700 to 500 . Subharmonic modes are indicated by filled circles and the dotted line for $|\mu|=1$ is the stability boundary.

prevailing for exercise waveforms at larger Reynolds numbers. In all calculations, the geometrical parameters have the values $W=0.5$ and $H=0.5$.

The absolute values of the Floquet multipliers associated with the first eight azimuthal modes of the flow perturbations are given in figure 23. All Floquet multipliers are real; the most dominant mode is $m=5$. Moreover, all modes with $m \geqslant 2$ can be classified as period-doubling, as indicated by the filled circles.

The azimuthal vorticity distributions of the perturbations are shown in figure 24 for various mode numbers. Here, the modes $m=1$ and $m=2$ are stable, and the modes $m=3, m=4$ and $m=5$ are unstable. The vorticity distributions associated with $m=6$, $m=7$ and $m=8$ are not shown, because they resemble closely that associated with mode $m=5$. It will be observed that in this case the perturbation fields associated with the stable modes $m=1$ and $m=2$ and the marginally unstable mode $m=3$ extend downstream of the dilatation, but that the perturbation fields associated with the higher-valued unstable modes are much more localized inside the dilatation; these more localized modes have the largest growth rate.

\section{Sensitivity to geometrical details}

During the tests that were conducted to validate our numerical tools we noticed a remarkable sensitivity of the results to details of the model geometry. Here, we take the opportunity to report these observations. We compare the results of numerical computations using the Gaussian shaped vessel of the present paper with results obtained for a sinusoidally shaped vessel wall, the model aneurysm studied in the recent work of Sheard \& Ryan (2008) and Sheard (2009). For this comparison the same value of $H$ is taken, while $W$ is chosen such that the areas traced out in an axial plane by the vessel walls are the same. The two geometries, with the 'wavelength' of the sinusoidally shaped wall denoted $L$, are shown in figure 25 .

The pulsatile flow rate waveform in this comparison is that used by Salsac et al. (2006) and Sheard (2009), which is called Q3 here and is shown in figure 26. 


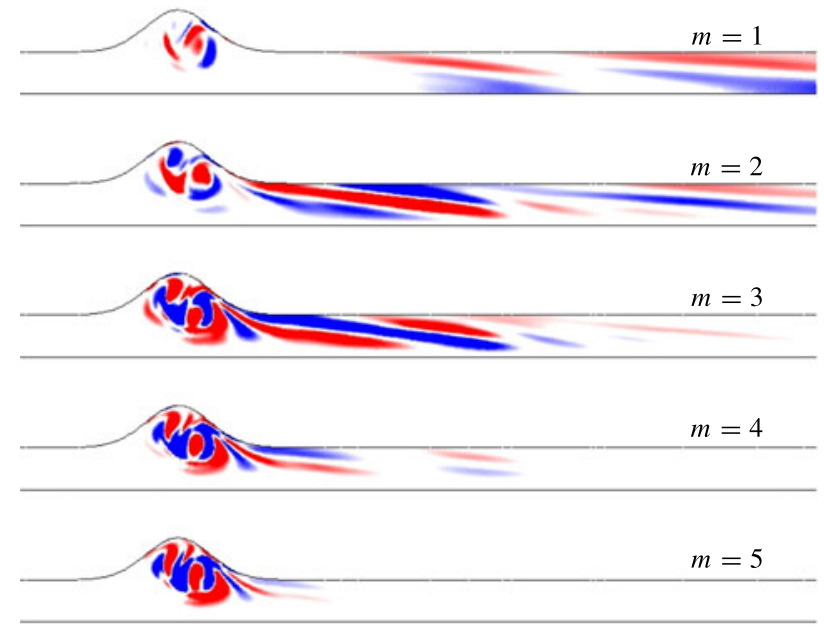

FIgURE 24. (Colour online) Azimuthal vorticity distributions of the perturbed flows associated with modes $m=1, \ldots, 5$ for pulsatile flow through a model aneurysm. The axial range shown is $-2.5 D \leqslant z \leqslant 10 D$ and the flow parameters and geometrical parameters have the values $R e=500, W o=13.8, W=0.5, H=0.5$. The Womersley number corresponds to the flow rate waveform $Q 2$ (exercise), but the Reynolds number is reduced from 1700 to 500 .

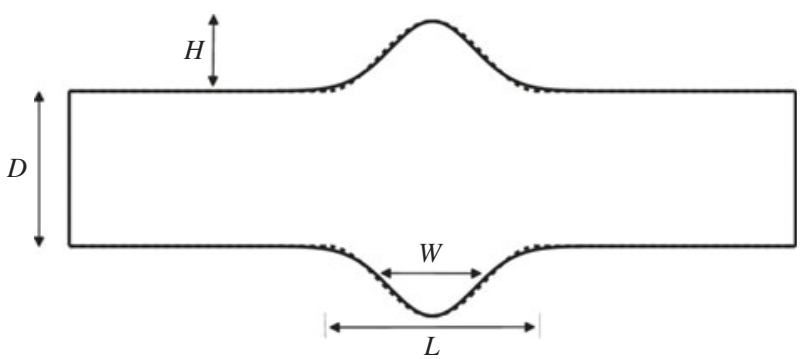

FIGURE 25. The sinusoidal geometry used in the studies by Sheard \& Ryan (2008) and Sheard (2009) is shown by the dotted curves and the Gaussian shaped geometry used in the present study is shown by the solid curves. Both model aneurysms have the same area in a plane through the symmetry axis. Parameter values: $H=0.45, W=0.58, L=2.9$.

The flow parameters have the values $R e=330$ and $W o=10.7$. An important difference between waveforms $Q 1$ and $Q 3$ is that much larger negative flow rates occur for $Q 3$.

Figure 27 presents the evolution of the azimuthal vorticity distribution during one pulse cycle of the pulsatile flow of $Q 3$ through a model aneurysm with sinusoidal shape $(a)$ and with Gaussian shape $(b)$. Qualitatively, the flow phenomena in the two geometries are similar, but closer inspection reveals that there is a slight time delay in the evolution of the vorticity distribution in the Gaussian shaped aneurysm with respect to that in the sinusoidally shaped aneurysm. This may be attributed to the fact that the sinusoidal wall, where it connects to the straight vessel, is not as well rounded-off as the vessel with the Gaussian shaped wall. As a result, flow separation occurs at a slightly earlier stage during systole. This phenomenon can be seen best by comparing the frames (ii) which correspond (roughly) to peak systole. 


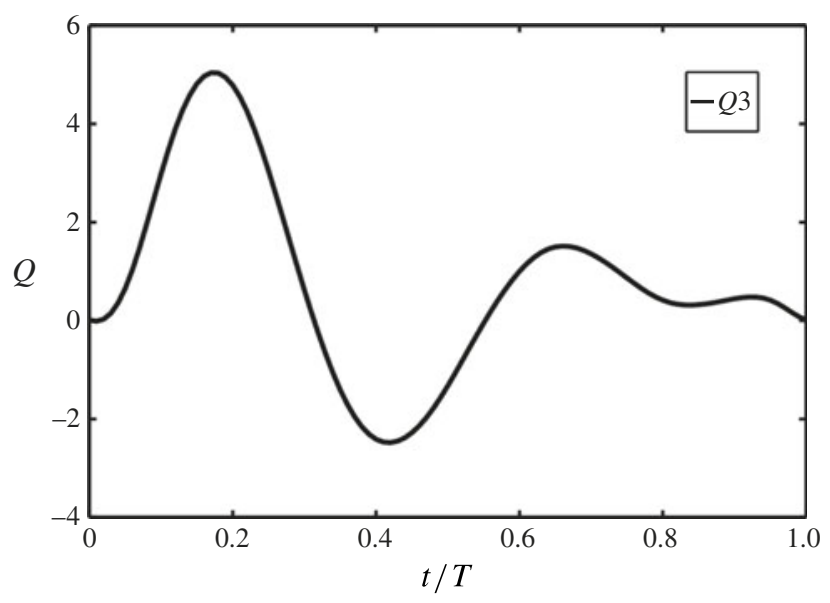

FIgURE 26. The waveform considered in the study by Salsac et al. (2006) and Sheard (2009).

(a)

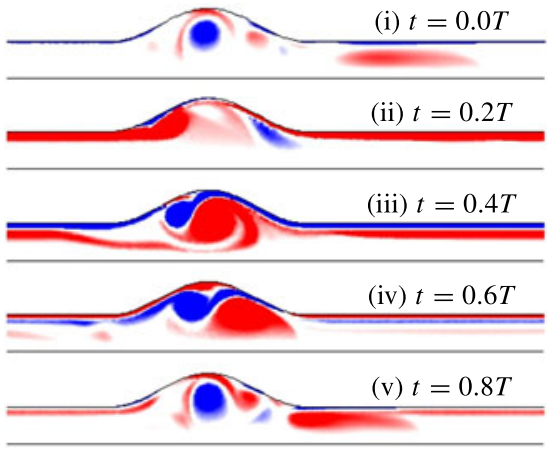

(b)

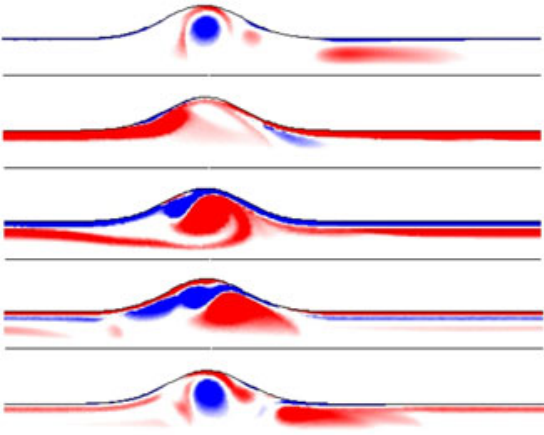

FIGURE 27. (Colour online) Evolution of the azimuthal vorticity distribution during one pulse cycle of the flow rate waveform $Q 3$ in aneurysms modelled by a sinusoidal $(a)$ and a Gaussian $(b)$ wall function. The dimensionless vorticity values range from -20 (blue) to +20 (red). The axial range is $-3 D \leqslant z \leqslant 5 D$. The values of the flow parameters and geometrical parameters are $R e=330, W o=10.7, H=0.45, W=0.58, L=2.9$.

The wall shear stress distribution has been found to be rather sensitive to the details of the vorticity distribution in an aneurysm, cf. $\S 3.2$, so that one would expect that the slight time delay just mentioned would be visible in plots of the wall shear stress. This is indeed the case. As figure 28 shows, the most prominent differences are found at the proximal and distal ends of the dilatation, i.e. close to the location where there is a difference in differentiability of the functions that describe the vessel wall shapes.

It will now not come as a surprise that the slight differences in the vorticity distributions of the pulsatile flows are also reflected in the stability characteristics of these flows. Figure 29 compares the absolute value of the Floquet multiplier associated with the azimuthal modes $m=1, \ldots, 5$ in the two geometries; here the flow parameters are $R e=330$ and $W o=10.7$. Qualitatively, the characteristics are the same: all Floquet multipliers are complex and in both cases the mode $m=3$ is the least stable mode; this was found also by Sheard \& Ryan (2008). The absolute 

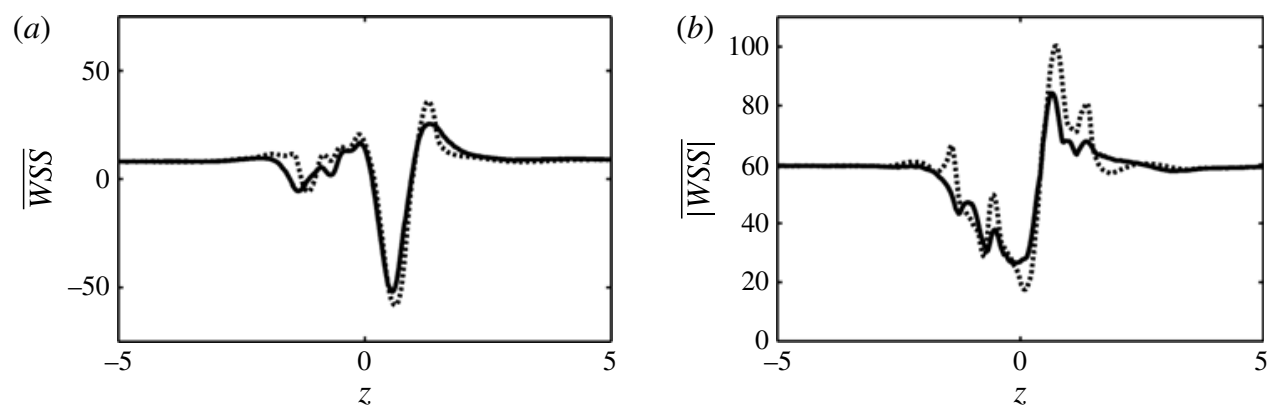

FIGURE 28. Axial variation of the cycle-averaged wall shear stress $\overline{W S S}(a)$ and cycle-averaged magnitude of the wall shear stress $\overline{|W S S|}(b)$ in aneurysms modelled by a sinusoidal (dotted curves) and a Gaussian (solid curves) wall function. The flow rate waveform is $Q 3$ and the values of the flow parameters and geometrical parameters are $R e=330, W o=10.7, H=0.45, W=0.58, L=2.9$.

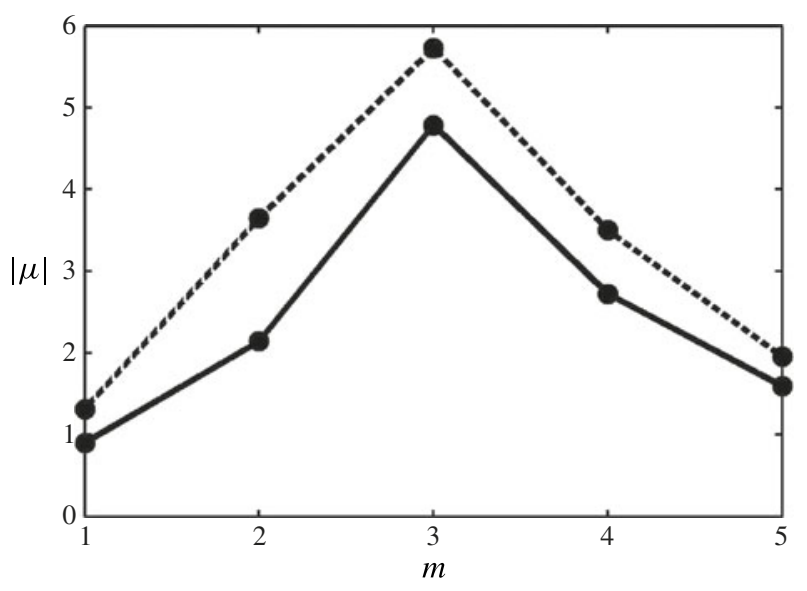

FIGURE 29. Variation of the absolute value of the Floquet multiplier $\mu$ with the azimuthal mode number $m$ for pulsatile flow through a model aneurysm with the wall described by a sinusoidal function (dotted curve) and a Gaussian function (solid curve). The flow parameters and geometrical parameters have the values $R e=330, W o=10.7, H=0.45$, $W=0.58, L=2.9$.

values of the Floquet multipliers, though, are significantly larger for pulsatile flow through the sinusoidally shaped aneurysm, the mode $m=1$ even being unstable while it is stable for the Gaussian shaped wall.

The azimuthal vorticity distributions of the perturbed flows associated with the various modes are shown in figure 30, for the sinusoidally shaped wall in $(a)$ and for the Gaussian shaped wall in $(b)$. Again, these vorticity distributions may seem similar; however, they are not indistinguishable. As found earlier, the higher mode numbers $m=2,3,4,5$ are localized within the dilatation, but the (most stable) mode $m=1$ extends far downstream.

This example shows that the details of pulsatile flow through aneurysms, the wall shear stress distribution in particular, can be extremely sensitive to geometrical details. The conclusion is that care must be taken when interpreting results from 
(a)

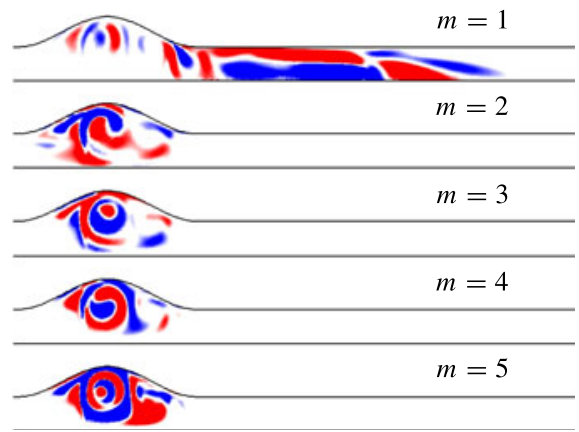

(b)

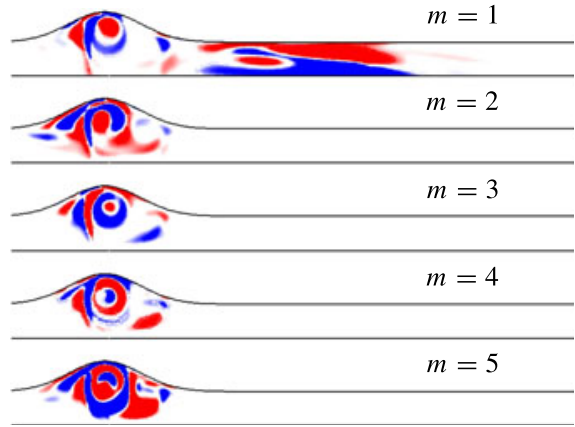

FIGURE 30. (Colour online) Azimuthal vorticity distributions of the perturbed flows associated with modes $m=1, \ldots, 5$ for pulsatile flow through a model aneurysm of sinusoidal shape $(a)$ and Gaussian shape $(b)$. The axial range shown is $-2.5 D \leqslant z \leqslant 10 D$. The flow rate waveform is $Q 3$, and the flow parameters and geometrical parameters have the values $R e=330, W o=10.7, H=0.45, W=0.58, L=2.9$.

('patient-specific') computations of the flow in an aneurysm geometry that is constructed from medical imaging data.

\section{Conclusions}

In this paper we have presented the results of numerical computations of pulsatile flow through a model abdominal aortic aneurysm, and of an analysis of the hydrodynamic stability of this flow. The aneurysm is modelled as a circular cylindrical vessel with a radius described by a Gaussian function. The flow at the inlet of the calculational domain is the Womersley solution for pulsatile flow in a cylindrical vessel. This solution is a Fourier-Bessel series; the coefficients of the series were deduced from measurements by Suh et al. (2011) of the blood flow rate waveform at the inlet of the infrarenal aorta, during rest and exercise conditions, of patients suffering from an abdominal aortic aneurysm.

For a given flow rate waveform, the problem is characterized by four dimensionless quantities: the Reynolds number $R e$ and Womersley number Wo of the fluid flow, and the 'height' $H$ and 'width' $W$ which specify the Gaussian geometry of the dilatation. In the present study, the Reynolds number was varied between 200 and 500 and the Womersley number between 10 and 15, for the flow rate waveform of the rest condition. The flow structure corresponding to an exercise condition was studied for $R e=1700$ and $W o=13.8$, but it turned out to be easier to use the value $R e=500$ in the stability analysis. To learn about the influence of the aneurysm geometry on the flow characteristics, the geometrical dimensionless quantities were varied in the ranges $0.3 \leqslant H \leqslant 1.0$ and $0.5 \leqslant W \leqslant 1.0$.

Typical flow phenomena that can be observed in pulsatile flow through an aneurysm are the creation of vorticity at the vessel wall during the acceleration phase of systole, followed by detachment of this wall-bounded shear layer at the proximal end of the dilation during the deceleration phase of systole; this leads to the formation of a cylindrical layer of concentrated vorticity with a radius comparable with that of the vessel upstream of the dilatation. For 'sufficiently localized' aneurysms, for Reynolds numbers and Womersley numbers in the physiological range, the shear layer rolls up and forms a ring-like vortex structure which interacts with the vessel wall and collides 
with it near the distal end of the dilatation. During this vortex-wall interaction, new vorticity of opposite sign is produced at the vessel wall; this eventually detaches and forms a secondary vortex ring with vorticity of opposite sign to that of the primary vortex ring. During the final stages of diastole, the primary vortex is washed downstream with the flow, while the secondary opposite signed vortex decays. This process then repeats in a new cardiac cycle. An increase of the Reynolds number and/or an increase in the height of the dilatation yields more vigorous vortex dynamics, in the sense that the vortices are more intense and approach the distal vessel wall at higher speed, and that further ring-like vortex structures are formed. The computations with the flow rate waveform corresponding to exercise conditions show that at high Reynolds number a highly complex vorticity distribution emerges.

This description of the flow phenomena in pulsatile flow through a Gaussian shaped model aneurysm agrees very well with what was found in experiments by Salsac et al. (2006). These authors also observed that the vortex motions within the dilatation induce strong spatial and temporal variations of the wall shear stress, especially near the distal end. Again, this agrees with what was found by us. Our computational approach allowed us to investigate a somewhat larger range of parameter values than Salsac et al. (2006). Our results support the conclusion of Salsac et al. (2006) that longer aneurysms are less pathological than short ones. Moreover, our results suggest that physical activity is harmful for a patient with an abdominal aortic aneurysm; the more-localized or well-developed aneurysms again being the most pathological.

Yip \& Yu (2001) and Salsac et al. (2006) mention that during part of the cardiac cycle the flow in an aneurysm may become weakly turbulent. Since this repetitive occurrence of disturbed flow conditions seems especially harmful, we have investigated the hydrodynamic stability of pulsatile flows through a model aneurysm. It appears that such flows are unstable to small-amplitude three-dimensional disturbances for flow conditions within the physiological range, flows through well-developed aneurysms being the most susceptible. Our findings support the idea that patients suffering from abdominal aortic aneurysms might want to avoid physical exercise. A second important observation is that the vorticity structures associated with the flow disturbances are not confined to the dilatation, but rather extend far downstream. The abdominal aortic aneurysm acts as a 'wavemaker' which generates disturbed flow conditions in the healthy section of the arterial system downstream of the aortic aneurysm. This may be related to the fact that one-fifth of larger abdominal aortic aneurysms are accompanied by an aneurysm in one or both of the common iliac arteries (Armon et al. 1998). It also leads to the conclusion that patient-specific computational studies of the biomechanics of abdominal aortic aneurysms should include the iliac bifurcation and the common iliac arteries as part of the computational domain.

Finally, during various numerical tests we observed a remarkable sensitivity of the flow to geometrical details of the model aneurysm. This became clear by carrying out the calculations of Sheard (2009), who modelled the aneurysm wall by a sinusoidal function, using a Gaussian function with the same maximal vessel radius and which encloses the same area in a plane through the axis of symmetry. The volume flow rate waveform was that used by Salsac et al. (2006) and Sheard (2009), obtained from a healthy male subject at rest, and so were the dimensionless flow quantities: $R e=330$ and $W o=10.7$. What was observed was that the exceedingly small difference in the differentiability of the shape functions, where the sinusoidal dilatation connects to the inlet vessel, leads to small, but still significant, differences in the wall shear stress distribution and the growth rate of three-dimensional flow perturbations. If the aim is 
to make a quantitative analysis through a patient-specific computational study it seems wise to carry out a sensitivity analysis to assess the influence of unavoidable minor errors that occur when transforming medical images into a computational domain.

\section{Acknowledgements}

The authors wish to express their gratitude to Professor P. Feugier of the Université Claude-Bernard Lyon 1, and vascular surgeon at Hôpital Édouard-Herriot in Lyon, for many useful discussions.

\section{REFERENCES}

Armon, M. P., Wenham, P. W., Whitaker, S. C., Gregson, R. H. S. \& Hopkinson, B. R. 1998 Common iliac artery aneurysms in patients with abdominal aortic aneurysms. Eur. J. Vasc. Endovasc. Surg. 15, 255-257.

BARAKAT, A. I. 2013 Blood flow and arterial endothelial dysfunction: mechanisms and implications. C. R. Phys. 14, 479-496.

Deplano, V., Knapp, Y., Bertrand, E. \& Gaillard, E. 2007 Flow behaviour in an asymmetric compliant experimental model for abdominal aortic aneurysm. J. Biomech. 40, 2406-2413.

Egelhoff, C. J., Budwig, R. S., Elger, D. F., Khraishi, T. A. \& Johansen, K. H. 1999 Model studies of the flow in abdominal aortic aneurysms during resting and exercise conditions. Biorheology 32, 1319-1329.

Finol, E. A., Keyhani, K. \& Amon, C. H. 2002 The effect of asymmetry in abdominal aortic aneurysms under physiologically realistic pulsatile flow conditions. Trans. ASME J. Biomech. Engng 125, 207-217.

GopAlakrishnAN, S. S. 2014 Dynamics and stability of flow through an abdominal aortic aneurysm. $\mathrm{PhD}$ thesis, Université de Lyon.

Gopalakrishnan, S. S., Pier, B. \& Biesheuvel, A. 2014 Global stability analysis of flow through a fusiform aneurysm: steady flows. J. Fluid Mech. 752, 90-106.

Griffith, M. D. 2007 Stabilité et dynamique des écoulements en géométrie de sténose. PhD thesis, Université de Provence, Aix-Marseille and Monash University, Melbourne.

Griffith, M. D., Leweke, T., Thompson, M. C. \& Hourigan, K. 2009 Pulsatile flow in stenotic geometries: flow behaviour and stability. J. Fluid Mech. 622, 291-320.

Grootenboer, N., Bosch, J. L., Hendriks, J. M. \& VAn Sambeek, M. R. H. M. 2009 Epidemiology, aetiology, risk of rupture and treatment of abdominal aortic aneurysms. Eur. J. Vasc. Endovasc. Surg. 38, 278-284.

Harter, L. P., Gross, B. H., Callen, P. W. \& Barth, R. A. 1982 Ultrasonic evaluation of abdominal aortic thrombus. J. Ultrasound Med. 1, 315-318.

Herbert, T. 1988 Secondary instability of boundary layers. Annu. Rev. Fluid Mech. 20, $487-526$.

Humphrey, J. D. \& Holzapfel, G. A. 2012 Mechanics, mechanobiology, and modeling of human abdominal aorta and aneurysms. J. Biomech. 45, 805-814.

Humphrey, J. D. \& TAYloR, C. A. 2008 Intracranial and abdominal aortic aneurysms: similarities, differences, and need for a new class of computational models. Annu. Rev. Biomed. Engng 10, 221-246.

Johnston, K. W., Rutherford, R. B., Tilson, M. D., Shah, D. M., Hollier, L. \& StAnley, J. C. 1991 Suggested standards for reporting on arterial aneurysms. J. Vascu. Surg. 13, 452-458.

Ku, D. N. 1997 Blood flow in arteries. Annu. Rev. Fluid Mech. 29, 399-434.

LAsheras, J. C. 2007 The biomechanics of arterial aneurysms. Annu. Rev. Fluid Mech. 39, $293-319$.

Les, A. S., Shadden, S. C., Figueroa, C. A., Park, J. M., Tedesco, M. M., HerfKens, R. J., DAlman, R. L. \& TAYlor, C. A. 2010 Quantification of hemodynamics in abdominal aortic aneurysms during rest and exercise using magnetic resonance imaging and computational fluid dynamics. Ann. Biomed. Engng 38, 1288-1313. 
Pedley, T. J. 1979 The Fluid Mechanics of Large Blood Vessels. Cambridge University Press.

Reininger, A. J., Heinzmann, U., Reininger, C. B., Friedrich, P. \& Wurzinger, L. J. 1994 Flow-mediated fibrin thrombus formation in an endothelium-lined model of arterial branching. Thrombosis Res. 74 (6), 629-641.

Robichaux, J., Balachandar, S. \& VAnKa, S. P. 1999 Three-dimensional Floquet instability of the wake of a square cylinder. Phys. Fluids 11, 560-578.

SAlsAC, A. V. 2005 Évolution des contraintes hémodynamiques lors de la croissance des anévrismes aortiques abdominaux. PhD thesis, École Polytechnique.

Salsac, A. V., Sparks, S. R., Chomaz, J. M. \& Lasheras, J. C. 2006 Evolution of the wall shear stresses during the progressive enlargement of symmetric abdominal aortic aneurysms. J. Fluid Mech. 560, 19-51.

Salsac, A. V., Sparks, S. R. \& Lasheras, J. C. 2004 Hemodynamic changes occurring during the progressive enlargement of abdominal aortic aneurysms. Ann. Vascu. Surg. 18, 14-21.

SHEARD, G. J. 2009 Flow dynamics and wall shear stress variation in a fusiform aneurysm. J. Engng Maths 64, 379-390.

ShEARD, G. J. \& RYAN, K. 2008 Wall shear stress and flow stability in a model fusiform aneurysm. ANZIAM J. 50, C1-C15.

Sheard, G. J., Thompson, M. C. \& Hourigan, K. 2005 Subharmonic mechanism of the mode C instability. Phys. Fluids 17, 111702.

Sheidaei, A., Hunley, S. C., Zeinali-Davarani, S., Raguin, L. G. \& Baek, S. 2011 Simulation of abdominal aortic aneurysm growth with updating hemodynamic loads using a realistic geometry. Med. Engng Phys. 33, 80-88.

Sherwin, S. J. \& Blackburn, H. M. 2005 Three-dimensional instabilities and transition of steady and pulsatile axisymmetric stenotic flows. J. Fluid Mech. 533, 297-327.

Stamatopoulos, C., Mathioulakis, D. S., Papaharilaou, Y. \& Katsamouris, A. 2011 Experimental unsteady flow study in a patient-specific abdominal aortic aneurysm model. Exp. Fluids 50, 1695-1709.

Suh, G. Y., Les, A. S., Tenforde, A. S., Shadden, S. C., Spilker, R. L., Yeung, J. J., Cheng, C. P., Herfiens, R. J., Dalman, R. L. \& Taylor, C. A. 2011 Hemodynamic changes quantified in abdominal aortic aneurysms with increasing exercise intensity using MR exercise imaging and image-based computational fluid dynamics. Ann. Biomed. Engng 39, 2186-2202.

TAYlor, C. A. \& Draney, M. T. 2004 Experimental and computational methods in cardiovascular fluid mechanics. Annu. Rev. Fluid Mech. 36, 197-231.

TAYlor, C. A. \& FigueroA, C. A. 2009 Patient-specific modeling of cardiovascular mechanics. Annu. Rev. Biomed. Engng 11, 109-134.

TAYlor, C. A., Hughes, T. J. R. \& Zarins, C. K. 1999 Effect of exercise on hemodynamic conditions in the abdominal aorta. J. Vascu. Surg. 29, 1077-1089.

TAYlor, C. A. \& YAMAguchi, T. 1994 Three-dimensional simulation of blood flow in an abdominal aortic aneurysm - steady and unsteady flow cases. Trans. ASME J. Biomech. Engng 116, 89-97.

VORP, D. A. 2007 Biomechanics of abdominal aortic aneurysm. J. Biomech. 40, 1887-1902.

Vorp, D. A., Lee, P. C., Wang, D. H. J., Makaroun, M. S., Nemoto, E. M., OGaWA, S. \& Webster, M. W. 2001 Association of intraluminal thrombus in abdominal aortic aneurysm with local hypoxia and wall weakening. J. Vascu. Surg. 34, 291-299.

YIP, T. H. \& YU, S. C. M. 2001 Cyclic transition to turbulence in rigid abdominal aortic aneurysm models. Fluid Dyn. Res. 29, 81-113.

YIP, T. H. \& YU, S. C. M. 2002 Oscillatory flows in straight tubes with an axisymmetric bulge. Exp. Therm. Fluid Sci. 26, 947-961. 